\title{
Spatial-transcriptomics reveals unique defining molecular features of 5-aminolevulinic acid+ infiltrative tumor cells associated with glioblastoma recurrence and poor survival
}

\section{Geoffroy Andrieux}

University Medical Center Freiburg

\section{Tonmoy Das}

University of Dhaka

Michaela Griffin

Children's Brain Tumour Research Centre, Biodiscovery Institute, School of Medicine, University of Nottingham, Nottingham, UK

\section{Melanie Boerries}

Faculty of Medicine, Freiburg University, Germany

\section{Stuart Smith}

University of Nottingham https://orcid.org/0000-0002-4556-2707

\section{Ruman Rahman}

University of Nottingham https://orcid.org/0000-0002-6541-9983

Sajib Chakraborty ( $\nabla$ sajib@du.ac.bd)

University of Dhaka https://orcid.org/0000-0002-8900-503X

\section{Article}

Keywords: GBM, 5ALA, Spatial-transcriptomics, Mesenchymal subtype, Wound response, Stemnesssignature

Posted Date: August 4th, 2021

DOl: https://doi.org/10.21203/rs.3.rs-780275/v1

License: (c) (i) This work is licensed under a Creative Commons Attribution 4.0 International License. Read Full License 


\section{Title: Spatial-transcriptomics reveals unique defining molecular 2 features of 5-aminolevulinic acid+ infiltrative tumor cells associated 3 with glioblastoma recurrence and poor survival}

4 5

Geoffroy Andrieux ${ }^{1,2 \#}$, Tonmoy Das ${ }^{3 \#}$, Michaela Griffin ${ }^{4}$, Melanie Boerries ${ }^{1,2}$, Stuart J. Smith ${ }^{4}$, Ruman Rahman ${ }^{4^{*}} \&$ Sajib Chakraborty ${ }^{3^{*}}$

1. Institute of Medical Bioinformatics and Systems Medicine, Medical Center - University of Freiburg Faculty of Medicine, University of Freiburg, Freiburg, Germany.

2. German Cancer Consortium (DKTK) Partner Site Freiburg, German Cancer Research Center (DKFZ), Heidelberg, Germany.

3. Molecular systems biology laboratory, Department of Biochemistry and Molecular Biology, University of Dhaka, Dhaka, Bangladesh

4. Children's Brain Tumour Research Centre, Biodiscovery Institute, School of Medicine, University of Nottingham, Nottingham, UK

$$
\text { \# authors contributed equally }
$$

${ }^{*}$ Corresponding author(s)

\section{Sajib Chakraborty}

Molecular Systems Biology Laboratory

Department of Biochemistry and Molecular Biology

Faculty of Biological Sciences

University of Dhaka, Dhaka-1000, Bangladesh

sajib@du.ac.bd

${ }^{*}$ Corresponding author(s)

Ruman Rahman

Children's Brain Tumour Research Centre

Biodiscovery Institute

School of Medicine

University of Nottingham, Nottingham, UK

Ruman.Rahman@nottingham.ac.uk 


\section{Abstract}

Spatiotemporal-heterogeneity originating from genomic and transcriptional variation contributes to subtype switching in GBM prior to and upon recurrence. Fluorescenceguided neurosurgical resection utilizing 5-aminolevulinic acid (5ALA) enables the isolation of infiltrative margin tumor cells $(5 A L A+)$ from a non-neoplastic background within neighboring brain parenchyma. Spatial transcriptomics identifies GBM molecular subtype plasticity as not restricted to recurrence, but manifesting regionally in a celltype-specific manner, where a 5ALA+ Mesenchymal subtype may drive recurrence. Exon-intron split analysis reveals hijacking of the neural wound response pathway to promote tumor growth, via IRF8-mediated post-transcriptonal control. A unique stemness index further defines 5ALA+ cells as a rare sub-population of infiltrative GBM stem cells. Finally, we establish that enriched gene signatures of 5ALA+ cells are associated with poor survival and recurrence in GBM, signifying that transition from primary to recurrent GBM is not discreet, but rather a continuum whereby 5ALA+ residual disease more closely resembles the eventual recurrent GBM.

Keywords: GBM, 5ALA, Spatial-transcriptomics, Mesenchymal subtype, Woundresponse, Stemness-signature, 


\section{Introduction}

69 Isocitrate dehydrogenase [IDH]-wild-type Glioblastoma (GBM) is a highly aggressive and heterogeneous tumor with poor survival outcome. Despite radical multimodal treatment of aggressive surgery, radiation therapy, and chemotherapy with

72 temozolomide, the poor treatment outcome of GBM patients has remained stagnant with a median survival of 14.6 months from diagnosis ${ }^{1}$. One of the key reasons of poor treatment response is the invasiveness of GBM deep into the neighboring brain parenchyma, which renders complete surgical resection impossible and efficacious brain penetration of chemotherapeutics a considerable challenge ${ }^{2}$. Furthermore, the ineffectiveness of therapeutic agents may arise from the plasticity of GBM cells which manifests intra- and inter-tumor heterogeneity; indeed, failed molecular targeted therapeutics have historically been focused on the GBM proliferative genotype ${ }^{3}$. Heterogeneity in GBM has now been well established and contributes to the differential expression of subclonal genes owing to distinct molecular events developing during the spatiotemporal evolutionary lifespan of GBM cells ${ }^{4}$.

In search of the potential origins of phenotypic diversity and plasticity of GBM cells, emerging evidence indicates the existence of a rare GBM stem cell (GSC) subpopulation with self-renewing capacity ${ }^{5,6}$. Major characteristics of the GSC include hijacking the normal neural stem cell developmental programs to promote and maintain tumor growth, and acquisition of mechanisms to resist chemotherapy ${ }^{7}$. A recent singlecell RNA sequencing (scRNA-seq) study revealed high inter- and intra-GSC distinct gene signatures of two cellular states - 'normal neural development' and 'inflammatory wound response' and concluded that a transcriptional program resembling a neural wound response in GSC may functionally contribute to GBM initiation ${ }^{8}$. To gain insight into the functional developmental and metabolic programs of GBM cells, Garofano et al. integrated scRNA-seq and bulk transcriptomics data by using a computational platform (single-cell biological pathway deconvolution (scBiPaD) and showed that the distribution of GBM cells along neurodevelopmental and metabolic 
axes, could facilitate their classification as proliferative/progenitor, neuronal, mitochondrial (MTC) and glycolytic/plurimetabolic (GMP) subtypes ${ }^{9}$. Based on transcriptional attributes, GBM was originally classified into four subtypes: Classical (CL), Neural (NE), Proneural (PN), and Mesenchymal (MES) subtypes ${ }^{10}$. However, a more recent study based on GBM transcriptomics analysis, excluding nonmalignant cell types, confirmed three subtypes of GBM - CL, PN, and MES ${ }^{11}$, but where most patients exhibit a plasticity of varying subtypes within the same tumor ${ }^{4}$. Moreover, longitudinal studies demonstrated the temporal plasticity of GBM subtypes by uncovering the subtype switch in GBM patients upon recurrence ${ }^{11,12}$. Recently, Minata et al. showed that in response to the radiation-induced proinflammatory microenvironment, GBM cells at the tumor edge acquire a MES subtype defined by the expression of CD109 ${ }^{13}$.

In addition to temporal plasticity, a growing body of evidence suggests that a collection of tissue from a single site fails to capture the spatial heterogeneity and transcriptional dynamics of GBM cells ${ }^{14}$. Tissues isolated from the invasive tumor margin (herein referred to as 'Inv') where GBM penetrates into the normal brain parenchyma, harbors distinct genomic and transcriptomic profiles in contrast to tissue removed from tumor core (herein referred to as 'Core') and enhancing rim (herein referred to as 'Rim') regions ${ }^{14}$. As recurrence of GBM is initiated within and beyond $2 \mathrm{~cm}$ of the Inv postsurgery, ${ }^{15}$ unique molecular features which characterize this region maybe targeted to impair the recurrence of GBM. Nevertheless, the highly heterogeneous cellularity of tissues taken from the Inv, including infiltrated immune and normal neural cells, pose a substantial challenge to filter the tumor-specific genomic and transcriptomic profiles from an overwhelming background of non-neoplastic cells.

A viable solution emerged from the use of 5-aminolevulinic acid (5ALA) during GBM neurosurgery ${ }^{16}$. 5ALA - a porphyrin - is metabolized to the fluorescent metabolite protoporphyrin IX (PplX), by cells in which the heme biosynthetic pathway is activated, such as GBM cells but not non-neoplastic cells ${ }^{14}$. GBM cells exhibit a marked accumulation of PpIX due to an inability to incorporate iron into the protoporphyrin core 
129 due to the absence of ferrochelatase enzyme activity ${ }^{14}$. The necrotic tumor core does not emit fluorescence owing to its deficiency of active heme metabolism; in contrast, the invasive tumor margin fluoresces brightly and eventually fades with the decreasing number of tumor cells in the periphery ${ }^{17}$. We previously have shown that invasive regions from primary tissue harboring the infiltrative 5ALA+ tumor cells can be purified from background non-neoplastic cells through fluorescence-activated cell sorting (FACS) ${ }^{14}$.

Intra-tumor surgical sampling, aided by FACS-isolation of 5ALA+ and 5ALAsubpopulations from invasive margin tissue, can serve as an ideal resource to untangle the intricacy of cellular and transcriptional heterogeneity within GBM. We have demonstrated that analysis of spatial transcriptomics (ST) within this sampling framework can identify non-canonical molecular factors associated with GBM infiltration, such as SERPINE1 ${ }^{14}$. However, deep profiling of transcriptomic landscapes within 5ALA sorted cells, is required to elucidate the spatial transcriptional heterogeneity and unique molecular signatures of 5ALA+ cells. Since 5ALA+ cells represent a rare tumor subpopulation within the Inv and importantly, are in closest proximity to residual disease remaining post-surgery, it is pertinent to explore the molecular subtypes, cellular states, and immune-related pathways in 5ALA+ cells and how this differs from 5ALA- nonneoplastic cells and distinct intratumor regions of GBM. Elucidating molecular signatures of emerging cellular (developmental and inflammatory wound response) and metabolic (GPM and MTC) states in 5ALA+ cells, is crucial to better understand the role of these infiltrative cells in tumor promotion and maintenance. Moreover, interrogating this rare subpopulation for stem-cell like features can also shed light on survival outcome and recurrence of GBM.

To address these questions, we performed ST analyses of unsorted Core, Rim, and Inv tissue, in addition to FACS-isolated 5ALA+ and 5ALA- cells across 10 GBM patients. We interrogated the transcriptomic landscape for molecular, cellular, metabolic and stemness gene signatures, to test the hypothesis that the 5ALA+ subpopulation(s) is defined by a unique cellular state. To understand the transcriptional programs of 5ALA+ 
cells, we employed exon-intron split analysis (EISA) ${ }^{18}$ to distinguish between transcriptional and post-transcriptional control mechanisms and ARACNE (Algorithm for the Reconstruction of Accurate Cellular Networks) algorithm to construct the transcriptional network governing unique gene signatures of $5 A L A+$ cells. Lastly, we explored the association of 5ALA+ cells with survival outcome and recurrence of GBM.

\section{Results:}

Correlation analysis reveals transcriptome compatibility of unsorted GBM tissues and FACS-isolated cells

RNA-seq data from tissue samples representing spatially distinct regions (Core, Rim, Inv) of tumor samples across $10 \mathrm{GBM}$ patients were analyzed. Neuropathological and molecular analyses confirmed that all 10 patients had GBM with wild-type isocitrate dehydrogenase 1 (IDH1) and transcriptional regulator - ATRX (Supplementary Table S1). All patients were subjected to surgical resection of the tumor aided by 5ALA immunofluorescence guidance. Cells from the Inv were dissociated and subjected to FACS isolation based on 5ALA immunofluorescence. Due to the distinct nature of the unsorted tissue and sorted cells (i.e. FACS process in the latter), we performed quality control analysis to evaluate the compatibility of the transcriptome between unsorted tissues - Core, Rim and Inv - and sorted cells (5ALA+ and 5ALA-). The analysis showed that the $\log _{2}$ normalized RNA-seq counts $(T P M+1)$ were uniform and showed less variance across different patients in the unsorted regions. Inter-region variability did not supersede the intra-region patient variability in terms of mRNA-expression. However, a notable variation was observed between the 5ALA sorted cells and the unsorted tumor regions (Core, Rim, and Inv) (Supplementary Figure S1A). The underlying cause for these differences could be attributable to both biological and technical factors. The unsorted regions represent heterogeneous cell populations within the tissue, whereas the sorted cells were isolated and subjected to FACS analysis. Moreover, the percentage of $5 A L A+$ cells of the total cell population in the Inv samples gated based on the 5ALA fluorescence, showed variation ranging from $0.9-2.90 \%$ with an average of $1.59 \%$ (Supplementary Table S1). The three patients with lower 5ALA+ transcriptome levels had a slightly lower average 5ALA+ cell percentage (1.50\%) compared to the rest 
191 of the samples (1.63\%). To evaluate the compatibility further, we calculated the Pearson

192 correlation coefficients on the normalized mRNA expression data amongst the different 193 regions and 5ALA sorted cells. Analysis indicated that correlation values across 194 different tumor regions and cells were uniform ranging from 0.88 to 1.00 . When the 195 expression of shared genes were compared between unsorted regions and sorted cells, a reasonable correlation was observed (Core vs. 5ALA- : 0.92, Rim vs. 5ALA- : 0.93, Inv vs. 5ALA- : 0.91, Core vs. 5ALA+: 0.89, Rim vs. 5ALA+ : 0.89, and Inv vs. 5ALA+: 0.88) (Supplementary Figure S1B). A higher correlation was also observed between 5ALA- and 5ALA+ cells $(R=0.95)$ as predicted by a shared brain microenvironment. The highly correlated transcriptome of the unsorted tissue and sorted cells were 201 indicative of their compatibility.

202

203

Differential regulation of cancer-specific and metabolic pathways in spatially distinct GBM regions

205 Having established the compatibility of the ST datasets from unsorted tissues (Core, 206 Rim and Inv) and 5ALA sorted cells (5ALA+ and 5ALA-), we performed a diverse array 207 of computational analysis to identify unique defining molecular features of 5ALA+ cells 208 relative to unsorted tissues and 5ALA- cells (Figure 1A). To determine the cancerrelated and metabolic pathways which are associated with distinct regions of GBM (Core, Rim, Inv) and 5ALA sorted cells from the Inv, a GSEA was performed (Supplementary Table S2) for cancer-hallmarks. Core exhibited the highest number of enriched pathways including Epithelial-mesenchymal transition and Hypoxia, which had 213 the highest NES and the lowest adjusted p-values (Supplementary Table S2), followed 214 by TNF- $\alpha$ signaling via NFKB , Interferon- $\alpha$ response, Inflammatory response, E2F 215 targets, and IL-6/JAK/STAT3 signaling (Figure 1B). Akin to the Core, Epithelial216 mesenchymal transition and Hypoxia were highly enriched in the Rim (Figure 1B and 217 Supplementary Table S2). The Core was also enriched with pro-proliferative pathways 218 such as mitotic-spindle, G2M checkpoint, mTOCR1 signaling and E2F targets, whereas 219 only mitotic-spindle was enriched in Inv (Figure 1B). To investigate the distribution of 220 proliferative cells across spatially distinct GBM regions, Ki-67 immunohistochemistry 221 (IHC) was performed, revealing a high number of proliferative cells in the Core 
222 superficial medial region (Figure 1C) followed by anterior medial (Figure 1D) and tumor223 edge regions (Figure 1E). In contrast to the Core, the GBM Inv exhibited a lower 224 number of proliferative cells (Figure 1F). The higher number of enriched pro-proliferative 225 pathways in the Core, underlie the increased number of proliferative cells observed in 226 this region compared to the Inv. The enrichment of Glycolytic and Angiogenesis 227 pathways (Figure $1 \mathrm{~B}$ and Supplementary Figure S2A and B) in addition to the absence 228 of oxidative phosphorylation in the Core and Rim, supports a Warburg-like effect 229 induced by the hypoxic conditions in these regions (Figure 1B and Supplementary 230 Figure S2C and D). In contrast, Hypoxia was neither enriched in the unsorted Inv, nor $2315 \mathrm{ALA}+$ and 5ALA- sorted cells. Enrichment of Oxidative phosphorylation in Inv further 232 corroborated the evidence suggesting the GBM infiltrative margin represents a non233 hypoxic microenvironment (Figure 1B). CD-31 IHC also reinforced this finding by 234 showing that Core and Rim were highly vascularized (Figure $1 G$ and $H$ ), whereas no 235 vascularization was observed in Inv (Figure 1I). Interestingly, TNF- $\alpha$ signaling via NFkB 236 and Inflammatory response pathways were highly enriched in 5ALA+ cells as well as in 237 the Core and Rim (Figure 1B, Supplementary Figure S2E, and S2F and Supplementary 238 Table S2).

To further investigate these enriched pathways, the leading edge genes contributing to 241 the enrichment were identified for Hypoxia, Glycolysis, TNF- $\alpha$ signaling via NFkB, and 242 Inflammatory response. Several genes associated with TNF- $\alpha$ signaling via NFKB, and 243 Inflammatory response pathways showed an overlap; thus these two pathways were 244 merged and common genes retained (Supplementary Table S2). A hierarchical 245 clustering algorithm (Euclidian distance) was applied to the leading edge genes 246 associated with Hypoxia, Glycolysis, and Inflammatory response/TNF- $\alpha$ signaling via 247 NFкB pathways (Supplementary Table S2), followed by the visualization of normalized 248 (z-scored) gene expression across different regions of 10 GBM patients as heatmaps 249 (Figure $1 \mathrm{~J}-\mathrm{K}$ and Supplementary Figure S2G). Differential expression of these genes 250 revealed that inter-tumor Core and Rim regions showed significantly higher relative 251 expression of hypoxia-response related genes including HMOX1, TGFBI, PDK1, 252 VEGFA, and LOX (Figure 1J). In contrast, both the unsorted Inv and sorted Inv cells 
253 (5ALA+ and 5ALA-) showed significantly lower relative expression of Hypoxia-response 254 genes (Figure 1J). A Kruskal-Wallis test confirmed the Hypoxia-associated gene expression as significantly upregulated in tumor-core compared to Inv, 5ALA+, and 5ALA- cells (Supplementary Figure $\mathrm{S} 2 \mathrm{H}$ ). Similarly, genes associated with Glycolysis showed comparable differential regulation and were significantly upregulated in the Core and Rim relative to the Inv, 5ALA+, and 5ALA- cells (Supplementary Figure S2G and S2I). In contrast, TNF- $\alpha$ signaling via NFKB, and Inflammatory response genes including IL1B, NFKBIA, IL6, PTGS2, CCL20, CCL2, CXCL3, SOCS3, and CXCL2 exhibited significantly higher expression was in 5ALA+ and 5ALA-cells relative to all unsorted tumor regions (Figure $1 \mathrm{~K}$ and Supplementary Figure S2J).

Next, we classified neural cell types to different GBM regions through enrichment analysis of gene signatures representing four neural cell types - Oligodendrocytes, Neurons, Astrocytes, and Cultured Astroglia (Supplementary Table S3). Core and Rim were enriched with all four cell types representing a heterogeneous cell population, whereas Inv was mostly enriched with the Neuronal cell type (Supplementary Figure S2K). Akin to Inv, 5ALA- cells were also enriched with the Neuronal cell type (Supplementary Figure S2K). Interestingly, no enriched Neural cell type signature was identified for 5ALA+ cells, indicating a likely evolution to a unique or hybrid cell-type signature that cannot be defined using canonical neural classifiers.

In summary, GSEA followed by hierarchical clustering revealed differential regulation of cancer-related and metabolic pathways in distinct GBM intra-tumor regions, suggesting adaptation to different microenvironmental selection pressures. 5ALA+ cells exhibited an upregulation of TNF- $\alpha$ signaling via NFkB, and Inflammatory response pathways, but could not be classified by any known Neural cell-type signatures.

Disparate subtype enrichment within distinct GBM intra-tumor regions and 5ALA sorted cells

To further characterize spatially both distinct GBM regions and 5ALA sorted cells, we utilized enrichment analysis to identify GBM molecular subtypes (CL, NE, PN, and 
284

285

286

287

288

289

290

291

292

293

294

295

296

297

298

299

300

301

302

303

304

305

306

307

308

309

310

311

312

313

MES) previously described by Verhaak et al. ${ }^{10}$ (Supplementary Table S4). A differential pattern of subtype enrichment was observed in spatially distinct GBM regions and 5ALA sorted cells; for example, Core and Rim regions were mostly enriched with $C L$ and $P N$ subtypes (Figure $2 A$ and Supplementary Figure S3A and B), whereas the NE subtype was highly enriched in the Inv region (Figure $2 \mathrm{~A}$ and Supplementary Figure S3C). 5ALA- cells were mostly associated with the NE and PN (Figure 2A and Supplementary Figure S3D). In contrast, 5ALA+ cells were highly enriched with the MES subtype (Figure $2 A$ ) with a high NES (2.10) and low adjusted $p$ value $\left(2.3 \times 10^{-6}\right)$ (Figure $2 \mathrm{~B}$ ). Moreover, the enrichment of the MES subtype is unique to 5ALA+ cells compared to all other regions and 5ALA- cells. To validate the cellular composition, IHC was performed to determine expression of NeuN, representing the proportion of neuronal cells in Inv relative to Core and Rim. Results showed that Core showed the lowest proportion of neurons, compared to Inv (Figure 2C and Supplementary Figure S3E). In contrast, Rim exhibited a higher proportion of neurons compared to Core (Figure 2D and Supplementary Figure S3E). The highest proportion of neurons was observed in Inv further corroborating the spatial molecular signatures (Figure 2E and Supplementary Figure S3E).

To gain a deeper insight into the differential expression of subtype-specific genes, we identified the leading edge genes that contributed significantly to the enrichment of a subtype for a specific GBM region/cellular population (Supplementary Table S4). Hierarchical clustering analysis of the leading edge genes showed that CL- and PNspecific genes were highly expressed in Core, Rim, and Inv relative to 5ALA+ cells (Figure 2F and Supplementary Figure S3F), whereas, Inv uniquely showed a higher expression of NE-specific genes, relative to all other regions and 5ALA sorted cells (Figure 2G, H and Supplementary Figure S3G and S3H). MES-specific genes exhibited differential expression with relatively higher expression in the 5ALA+ cells (Figure $2 \mathrm{I}$ and Supplementary Figure S3I). Interestingly, patient-specific differential expression was observed in 5ALA+ cells for MES-specific genes. For example, 3/10 patients with variable expression patterns remained distinct whilst the remaining $7 / 10$ patients were 
314 grouped into two separate clusters; the largest cluster comprising four patients showed 315 the highest expression of MES-specific genes (Figure 2l).

316 Collectively, these results suggest that GBM molecular subtypes do not manifest 317 uniformly throughout spatially distinct regions, but rather may vary in a region-specific 318 manner. 5ALA+ cells were uniquely enriched with the MES subtype, with neither any intra-tumor region nor 5ALA- cells being enriched for MES.

320

321

322

323

324

325

326

327

328

329

330

331

332

333

334

335

336

337

338

339

340

341

342

343

344

\section{Inflammatory wound response, glycolytic/plurimetabolic, and mitochondrial gene} signatures are enriched in 5ALA+ cells

We next applied gene-signatures characterizing two recently described cellular states Developmental and Inflammatory wound response $-{ }^{8}$ and two metabolic states mitochondrial (MTC) and glycolytic/plurimetabolic (GPM) - ${ }^{9}$ of GBM cells and performed GSEA in spatially-distinct GBM regions and 5ALA sorted cells (Supplementary Table S5). The GPM subtype genes (Supplementary Table S1) showed a minimal overlap $(\mathrm{N}=2)$ with the classical Glycolysis hallmark category (Supplementary Table S2). The GPM subtype has been defined by a diverse array of metabolic activities including glycolysis/hypoxia, lipids, amino acids, steroids, and iron/sulfur metabolism but not mitochondrial/oxidative phosphorylation ${ }^{9}$. GSEA revealed a differential enrichment of these cellular and metabolic subtypes across GBM regions (Figure 3A). GPM and Developmental subtypes were enriched in Core and Rim, with only the Developmental subtype enriched in Inv. Both GPM and MTC states were enriched in 5ALA+ and 5ALAcells, indicating that similar metabolic states of 5ALA sorted cells may reflect a shared infiltrative margin microenvironment. Amongst the metabolic states, GPM enrichment score was higher in 5ALA+ cells whereas MTC enrichment score was higher in the 5ALA- cells (Figure $3 A$ ). Interestingly, Inflammatory wound response was uniquely enriched in 5ALA+ cells (Figure 3A). For 5ALA+ cells, the highest enrichment score was observed for GPM (Figure $3 \mathrm{~B}$ ), followed by MTC (Figure $3 \mathrm{C}$ ) and Inflammatory wound response (Figure 3D). The leading edge genes of these distinct cellular and metabolic states were identified from GSEA output (Supplementary Table S5) and subjected to clustering analysis. A higher expression of GPM-associated genes in the 5ALA+ cells was observed for $7 / 10$ patients (Figure 3E and Supplementary Figure S4A), whereas 
345 MTC genes were mostly upregulated in the 5ALA- cells (Figure 3F and Supplementary 346 Figure S4B). Most of the genes associated with Inflammatory wound response were highly upregulated in the 5ALA+ cells relative to other regions (5ALA+vs. Core: $p$ value $=0.005$ and 5ALA+ vs. Inv: $p$-value $=0.0006$ ) (Figure 3D, G and Supplementary Figure S4C). Although not significant, average expressions of Inflammatory wound response genes were higher in 5ALA+ cells compared to 5ALA- cells. (Supplementary

351 Figure S4C).

352 In summary, these results highlight the existence of differential cellular and metabolic subtypes throughout spatially distinct GBM regions and 5ALA sorted cells. Similar metabolic states in the 5ALA+ and 5ALA- non neoplastic cells were identified, whereas the enrichment of the Inflammatory wound response pathway was unique to the 5ALA+ infiltrative tumor subpopulation.

Identification of transcriptional networks controlling different cellular and metabolic states in 5ALA+ cells

In order to gain deeper insight into the transcriptional control regulating the cellular and metabolic gene-signatures enriched in 5ALA+ cells, we constructed a transcriptional network governing the leading edge genes of Inflammatory response, TNF- $\alpha$ signaling, MES, MTC, GPM, and Inflammatory wound response pathways. First, we identified TFs in the leading edge gene sets by using ENCODE TF annotation and in total, eight TFs were identified (KLF4, IRF8, NFKB 1, NFKB IA, EGR2, REL, FOS, and FOSL2). These TFs and all leading edge genes were used as hubs and target genes respectively in the ARACNE algorithm to identify the TF-target gene association significance. Only TFtarget gene associations with a p-value $<0.05$ were considered. Based on the ARACNE output, a TF-target gene network was constructed and visualized by the Cytoscape tool (Figure 4A and Supplementary Table S6). All TFs (with the exception of FOS) showed a positive $\log _{2}$ fold change (FC) in 5ALA+ compared to 5ALA- cells, and amongst the positively regulated TFs, REL, IRF8, and NFKB 1 exhibited the highest FCs. Most of the

373 target genes (nodes) were also upregulated in 5ALA+ cells (Figure 4A). Amongst the 374 TFs, FOSL2 showed the highest number $(\mathrm{N}=103)$ of interaction (edges) with nodes, 375 followed by IRF8 $(\mathrm{N}=100)$ and $\mathrm{NFKB} 1(\mathrm{~N}=85)$. The lowest number of target gene 
376 interactions was identified for FOS $(N=35)$ whilst all other TFs exhibited higher $(>70)$

377 target gene interactions. For TNF- $\alpha$ signaling genes, the most prominent TF identified 378 was NFkBIA, controlling 22 genes, followed by EGR2 and KLF4, each controlling 21 379 genes (Figure 4B). The TFs controlling the highest number of Inflammatory wound 380 response genes were KLF4 $(\mathrm{N}=33)$, IRF8 $(\mathrm{N}=33)$, and NFkB1 $(\mathrm{N}=32)$. For the 381 Inflammatory pathway, the TFs controlling the most number of target genes were IRF8 $382(N=20)$, FOSL2 $(N=15)$ and REL $(N=14)$. The highest number of MES signature 383 genes were likely to be controlled by FOSL2 $(N=30)$, IRF8 $(N=29)$ and NFKB1 $(N=26)$, with FOLS2 also controlling seven MTC- and six GPM-associated genes. Eight GPM genes were identified to be controlled by NFkB1 and EGR2 (Figure 4B).

Overall, these results uncovered the transcriptional network controlling signature genes associated with enriched cellular and metabolic states in 5ALA+ infiltrative GBM cells. TNF- $\alpha$ signaling and Inflammatory wound response pathways are primarily controlled by the NFKBIA, EGR2, and KLF4 TFs, whereas the MES subtype is transcriptionally regulated by IRF8, NFkB1, and FOSL2. MTC and GPM subtypes were likely to be under transcriptional control of FOSL2 and NFKB1/EGR2, respectively.

Higher exon and intron counts of the 5ALA+ enriched subtypes uncover

\section{transcriptional and post-transcriptional regulation}

396 Exon-intron split analysis (EISA) was performed to determine the changes in pre-mRNA 397 (intron) and mature-mRNA (exon) counts across distinct GBM regions and 5ALA sorted cells. We performed differential expression analysis to identify genes with altered exon and intron counts in 5ALA+ cells relative to Core (Figure 5A), Rim (Figure 5B), and Inv regions (Figure 5C). Compared to unsorted regions, a higher number of genes with significant intronic changes ( $\Delta$ intron) was observed than genes with significant changes 402 in exon counts ( $\triangle$ exon) in $5 A L A+$ cells (Figure $5 A-C$ ) indicating a relatively strong transcriptional control regulating the genomic landscape of $5 A L A+$ cells. In contrast to unsorted regions, a lower number of genes with significant $\Delta$ exon $(N=140)$ and $\Delta$ intron $(\mathrm{N}=93$ ) counts were identified between 5ALA+vs. 5ALA- cells (Figure 5D). This lower 
underscores a similar transcriptomic landscape of these cells, which may be induced by a shared infiltrative margin microenvironment. However, the contribution of technical factors inducing the experimental processes involving the isolation and preparation of $5 A L A+/-$ cells for FACS analysis cannot be excluded.

To functionally characterize the DEGs with altered intron and exon counts, hallmark enrichment analysis was performed. Oxidative phosphorylation genes with higher exon counts were highly enriched in 5ALA+ cells relative to Core, Rim, and Inv regions (Supplementary Figure 5A). This result was in agreement with our previous results, showing enrichment of the MTC subtype in 5ALA+ cells (Figure 3A). Genes with higher intron counts in contrast, were mostly associated with UV response, Apical surface and KRAS signaling pathways in 5ALA+ cells (Supplementary Figure 5A). Furthermore, to investigate the transcriptional and post-transcriptional control of 5ALA+ cell-specific cellular/metabolic gene-signatures and TFs, we obtained $\Delta$ exon and $\Delta$ intron counts of the leading edge genes representing enriched gene-signatures and upregulated TFs between 5ALA+ cells relative to unsorted regions and 5ALA- cells. Upon ranking the genes of each signature based on the $\Delta$ exon and $\Delta$ intron values, we performed preranked GESA to investigate the enrichment of the gene-signatures (Supplementary Table S7). The results showed significant enrichment of the MES subtype, Inflammatory response and TNF- $\alpha$ signaling pathways, GPM subtype, and TFs genes with upregulated $\triangle$ exon counts in 5ALA+ cells (Figure 5E) relative to 5ALA-cells. In contrast, only Inflammatory response genes with upregulated $\Delta$ intron counts were enriched in 5ALA+ cells compared to 5ALA- cells (Figure 5E). Interestingly, a comparison of 5ALA+ cells with unsorted regions (Core, Rim, and Inv) mostly resulted in the enrichment of Inflammatory wound response, Inflammatory response, TNF- $\alpha$ signaling, and MES subtype with increased $\Delta$ intron counts (Figure $5 \mathrm{E}$ ). These pathways are likely to be transcriptionally restricted in 5ALA+ cells. TFs and GPM with upregulated $\triangle$ intron counts were enriched in 5ALA+ cells relative to both unsorted tumor regions and sorted 5ALAcells (Figure 5E) signifying transcriptional regulation controlling these genes.

Next, we identified significant DEGs with higher exon and intron counts in 5ALA+ cells. The highest number of DEGs were identified for wound response $(N=11)$ (Supplementary Figure S5B) followed by MTC $(\mathrm{N}=5)$ (Supplementary Figure S5C) and 
438 TNF- $\alpha$ signaling $(\mathrm{N}=4)$ (Supplementary Figure S5D). Only one DEG was identified for 439 the MES subtype (Supplementary Figure S5E) and Inflammatory response pathway 440 (Supplementary Figure S5F). For wound response, six genes (IER2, PLSJ, MOV10L1, 441 CCL2, MMP25, and ADAMTSL5) exhibited increased $\triangle$ exon and five genes (MMP19, 442 DMD, PLCXD3, MGLL, BTBD11) showed increased $\triangle$ intron in 5ALA+ cells 443 (Supplementary Figure S6). All DEGs associated with TNF- $\alpha$ signaling and MTC 444 (except PANX1) exhibited higher $\triangle$ exon counts in 5ALA+ cells (Supplementary Figure 445 S6). CCL2 associated with multiple subtypes - (Inflammatory wound response, 446 Inflammatory response, and TNF- $\alpha$ signaling) and showed significantly higher ( $p$ 447 value $<0.001$ ) $\Delta$ exon counts in 5ALA+ cells (Supplementary Figure S5D and S5F and 448 S6L). Collectively, these results decipher the transcriptional and post-transcriptional regulation of enriched cellular and metabolic gene-signatures in 5ALA+ infiltrative GBM.

Higher stemness profile and enriched gene-signatures of 5ALA+ cells may contribute to GBM recurrence

453 Estimation of a patient-specific stemness index based on mRNA expression (mRNAsi) 454 across spatially distinct GBM regions and 5ALA sorted cells was performed by the 455 method described by Malta et al. ${ }^{19}$. Hierarchical clustering (correlation-based) showed 456 distinct mRNAsi profiles for 5ALA sorted (5ALA+ and 5ALA-) cell populations and 457 unsorted Inv regions across 10 patients. Tumor Core and Rim regions exhibited similar 458 but low mRNAsi (Figure 6A). Interestingly, differential stemness profiles were observed 459 in a patient-specific manner. For instance, 5ALA+ cell populations of three patients 460 (sample 34, 31, and 30) exhibited a higher stemness index compared to the remaining 461 seven patients (Figure 6A). Similar differential stemness profiles across patients were 462 observed for 5ALA- cells and other unsorted regions. With the highest stemness profile, $4635 \mathrm{ALA}+$ cells showed a distinct stemness profile relative to all unsorted tumor regions 464 (Figure 6A and B). Although the stemness profile of 5ALA+ cells was higher than 5ALA465 cells and unsorted Inv tissue, the differences were not statistically significant (Figure 466 6B). However, 5ALA+ cells exhibit a significant higher stemness profile compared to 467 Core and Rim regions. Interestingly, stratification of TCGA-GBM samples (representing 
468 tumor Core) into molecular subtypes revealed a lower mRNAsi of GBM subtypes 469 compared to both 5ALA+ and 5ALA- cells (Figure 6B).

To identify the association between gene-signatures and the mRNAsi of the 5ALA+ 472 cells, we retrieved the stemness-associated genes (KLF4, MYC, CTNNB1, EPAS1, EZH2, KDM5B, NES, TWIST1, ABCG2, CD34, CD44, NANOG, PROM1, ZFP42, and ZSCAN4) as reported by Malta et al. ${ }^{19}$ and analyzed the correlation between mRNAsi and expression of these genes across the 10 patients (Supplementary Figure S7A). Only two genes - CD44 and ZSCAN4 - showed a positive correlation between mRNA expression and mRNAsi (Supplementary Figure S7A). Next, we obtained 13 known brain cancer and 13 stem cell markers from the cell marker database (http://biocc.hrbmu.edu.cn/CellMarker/help.jsp) ${ }^{20}$ and investigated the correlation between mRNAsi and mRNA expression. In this case, two genes - ITGA6, SLITRK6 and SOX9 - showed a positive correlation (Supplementary Figure S7B). However, when the expression levels of these genes were analyzed, CD44, ZSCAN4, SLITRK6, and SOX9 showed low expression in 5ALA+ cells (Supplementary Figure S7C and S7D).

To further characterize the stemness associated gene-signature, eight previously published stemness associated gene sets representing Consensus Stemness (Shaat et al.) ${ }^{21}$, Human embryonic stem cell - HuESC (Bhattacharya et al.) ${ }^{22}$, Stem cell (Palmer et al.) ${ }^{23}$, Myc induced genes (Kim et al.) ${ }^{24}$, Embryonic stem cell - ES1 (Ben-porath et al.), Sox2 induced genes (Ben-porath et al.) ${ }^{25}$, NANOG induced genes (Ben-porath et al.) ${ }^{25}$ and Epithelial Atypical squamous cells (ASC) (Richards et al.) ${ }^{8}$ were retrieved. GSEA showed that most of the gene-sets were highly enriched in the Core, followed by Rim and Inv regions. 5ALA- cells were enriched in Consensus Stemness, HuESC, and Myc gene sets. Interestingly, none of these gene-sets were enriched in 5ALA+ cells (Supplementary Figure S7E and Supplementary Table S8). These results all together underscored the uniqueness of the 5ALA+ cells implying that previously published canonical stemness-associated gene sets may not be accurate, nor informative, when 
Realizing the uniqueness of the 5ALA+ cells, we obtained the six gene-signatures (Inflammatory wound response, MES subtype, Inflammatory response, TNF-a signaling, GPM, and MTC) that were enriched in 5ALA+ cells, and performed the correlation analysis between stemness and mRNA expression of these genes. A total of 39 genes mostly associated with Inflammatory wound response, MES subtype, Inflammatory response, and TNF- $\alpha$ signaling pathways, showed a significant positive correlation with mRNAsi, and constitutes a holistic dataset, which we term 'GBM Infiltrative Stem' (Figure 6C and Supplementary Table S9). The highest correlation was observed for the gene - Transglutaminase 4 (TGM4; $\mathrm{R}=0.95)$. The impact of GBM Infiltrative Stem on survival of GBM patients from the TCGA cohort was investigated by a cox regression model (Supplementary Table S10). Three genes - Interleukin 6 (IL6), ATP Binding Cassette Subfamily A Member 13 (ABCA13), and Four and a half LIM domains protein

2 (FHL2) - were associated with poor survival outcome and a high correlation with mRNAsi (FHL2, $R=0.90$; IL6: $R=0.76$; and ABCA13, $R=0.77$ ) (Figure 6D and 6E). Overall, these findings encourage us to define GBM Infiltrative Stem as a 5ALA+ gene signature positively correlated with Inflammatory wound response, MES subtype, Inflammatory response and TNF-a signaling gene-signatures.

Enriched cellular and metabolic subtypes of 5ALA+ are associated with poor overall survival

519 Six 5ALA+ enriched cellular and metabolic gene-signatures (TNF-a signaling, Inflammatory response, MES subtype, Inflammatory response, GPM, and MTC) were subjected to univariate cox regression analysis by using TCGA-GBM patient survival

522 data followed by the Hazard ratios (HRs) calculation for individual genes. Genes with an $523 \mathrm{HR}$ of $>1$ and a p-value $<0.05$ were considered to be associated with risk 524 (Supplementary Table S10). A cox regression model revealed a significant risk 525 associated with genes defining all subtypes except MTC. The highest number of riskassociated genes were identified for the MES subtype ( $N=42$ ), followed by

527 Inflammatory wound response $(\mathrm{N}=30)$, TNF- $\alpha$ signaling $(\mathrm{N}=27)$ and Inflammatory 528 response $(\mathrm{N}=23)$ (Supplementary Table $\mathrm{S10}$ ). Chemokine CCL2 with significantly 529 higher exon count in 5ALA+ cells (Supplementary Figure 5D), exhibited a significant 
530 impact on the survival outcome of TCGA-GBM patients (HR: 1.1, min: 1 - max: 1.3, p531 value $=0.01)($ Supplementary Table 10).

532

533

534

535

536

537

538

539

540

541

542

543

544

545

546

547

548

549

550

551

552

553

554

555

556

557

558

559

560

To evaluate the combined impact of risk-associated genes on survival outcome, TCGAGBM patients were classified into high- and low-expression cohorts by using a median expression threshold for each subtype to determine overall survival (OS) (Figure 7 A-E) and disease-free survival (DFS) (Supplementary Figure S8A-G). Kaplan-Meier curves showed that all 5ALA+ enriched gene-signatures were associated with poor DFS, with the exception of the MTC subtype (Supplementary Figure S8A-G). MES (Figure 7C), inflammatory wound response (Figure 7D) and GPM (Figure 7E) were associated with poor OS, where the highest significance was observed for the MES subtype ( $p$-value = 0.0084). The highest significance (lowest LogRank $p$-value) for poor DFS outcome was associated with a high-expression group of Inflammatory response (Supplementary Figure 8B), followed by Inflammatory wound response (Supplementary Figure 8D) and MES (Supplementary Figure 8C) gene-signatures.

To explore the impact of the 5ALA+ enriched gene signatures on the recurrence of GBM, GSEA was performed on RNA-seq data obtained from primary and recurrent IDH wild-type patients of TCGA, CGGA and GLASS cohorts (Supplementary Table S11). First, a hallmark enrichment analysis between recurrent and primary samples revealed that most of the enriched pathways in the 5ALA+ subpopulation(s) were detected in recurrent tumors. Primary tumors only exhibited marginal enrichment of pathways such as Hypoxia, DNA repair, E2F targets, whereas a diverse array of pathways including Oxidative phosphorylation, MYC targets, Fatty acid metabolism, IL6/JAK/STAT signaling, Epithelial-Mesenchymal transition, TNF- $\alpha$ signaling via NFKB and Inflammatory response, were enriched at recurrence and where the two latter pathways were also enriched in 5ALA+ cells (Supplementary Figure S9). TCGA harbors an unequal sample distribution between primary $(N=154)$ and recurrent $(N=13)$ tumor samples. Among the thirteen recurrent GBM samples, six were paired with primary tumor data. Therefore paired and unpaired analyses were performed for TCGA (Supplementary Table S11). For CGGA and GLASS cohorts primary and recurrent 
561 tumor samples are unpaired and paired, respectively. Enrichment analysis revealed an 562 intriguing phenomenon where 5ALA+ gene-signature enrichment was observed in recurrent but not in primary tumors. For instance, MES and Inflammatory wound response were enriched in recurrent tumors across TCGA, CCGA and GLASS cohorts (Figure 7F). GBM Infiltrative Stem was enriched in recurrent GBM of TCGA and GLASS cohorts but not in CGGA (Figure 7F). Compared to recurrent tumors, only MTC gene signatures were marginally enriched in the primary tumors of TCGA (Figure 7F). To further investigate the impact of unique 5ALA+ gene-signatures on the survival of recurrent and primary GBM patients, single sample GSEA (SSGSEA) was performed on recurrent and primary tumors separately for two unique gene-signatures in 5ALA+ cells - MES and Inflammatory wound response. By combining the NES, a combined 5ALA+ gene-signature score was calculated for each tumor sample, followed by correlation analysis with survival data of recurrent and primary patients. For recurrent tumors of TCGA (Figure 7G) and GLASS (Figure 7I), 5ALA+ gene-signature scores exhibited a significant negative correlation with survival, whereas primary tumors did not show a significant correlation with survival (Figure $7 \mathrm{H}$ and $\mathrm{J}$ ).

In conclusion, the enriched gene signatures of 5ALA+ cells were associated with poor survival and recurrence in GBM, signifying the probable functional impact of these signatures in tumor progression and interval to disease recurrence (Figure 7l).

\section{Discussion}

We have revealed distinct cellular, stemness and metabolic gene-signatures in spatially delineated intra-tumor GBM regions and 5ALA fluorescence-sorted invasive margin cells. Enriched gene-signatures in 5ALA+ cells, including our newly defined GBM Infiltrative Stem, are uniquely associated with poor survival and tumor recurrence, implicating 5ALA+ cells as accurate proximates of GBM residual disease post-surgery.

Previously, it has been hypothesized that 5ALA fluorescence beyond the T1 enhancing region on magnetic resonance imaging $(\mathrm{MRI})$, represents a unique microenvironment contributing to molecular signatures distinct to tumor Core and $\mathrm{Rim}^{2}$. Corroborating this 
592 hypothesis, we showed that pro-proliferative pathways (e.g. G2M checkpoint, mTOCR1 593 signaling and E2F targets) were highly enriched in the Core but absent in the infiltrative margin (Figure 1B). Moreover, upregulation of Glycolytic and Angiogenesis pathways

595 596 coupled with an absence of Oxidative phosphorylation in the Core and Rim (Figure 1B) signifies a hypoxia-induced Warburg effect. In contrast, the Hypoxia-response pathway was absent and oxidative phosphorylation pathway was upregulated, in both the unsorted invasive margin, 5ALA+ and 5ALA- sorted cells, implying a lack of hypoxic pressure in the microenvironment harboring residual, infiltrative disease (Figure 1B). This encourages reconsideration of delivery carriers which are designed to trigger the release of therapeutic payloads only in low $\mathrm{pH} /$ reducing conditions.

5ALA+ cells showed a unique immune-system associated molecular signature including TNF- $\alpha$ signaling via NFKB and Inflammatory response (Figure $1 B$ and $3 A$ ) relative to 5ALA- cells. This corroborates with our previously reported upregulation of Inflammatory response and downregulation of Hypoxia response genes in 5ALA+ cells ${ }^{14}$. This refutes the notion that certain cellular states are artifactually established due to the process of FACS, and supports the claim that these states reflect the biology of infiltrative margin residual disease. Enrichment of the NeuN cell type in both unsorted Inv and 5ALAcells, indicates that normal neural cells constitute the majority of the infiltrative margin, consistent with previous findings ${ }^{26}$. One of the striking features of 5ALA+ cells was the lack of molecular identity to canonical neural cell types, underscoring the unique transcriptional landscape infiltrative GBM. Using a microarray-based study, Bonnin et al. also showed that 5ALA+ tumor tissue failed to exhibit molecular signatures of any known cell types ${ }^{27}$.

Emerging evidence supports molecular subtype plasticity and subtype switching to MES in particular, being associated with recurrence and poor survival outcome ${ }^{11,13}$. To extend this hypothesis we show that the plasticity of GBM molecular subtypes is not restricted to recurrence, but can manifest in a region and cell-type-specific manner. Unsorted Core and Rim were highly enriched with CL- and PN-subtypes (Figure 2A), and unsorted Inv enriched with NEU (Figure 2A). 5ALA+ cells in contrast, were uniquely 
623 enriched with the MES subtype (Figure 2B) supporting a hypothesis that an infiltrative 624 MES subtype may drive GBM recurrence. Interestingly, a link between a MES gene625 signature and decreased tumor purity has been established as a common theme across 626 different cancers ${ }^{28,29}$. One of the prominent features of MES is the association with 627 immune-related pathways and the lower purity score in comparison to PM and CL, 628 highlighting the possible infiltration of non-neoplastic and immune cells into MES GBM 11, 30. In agreement with our previous results ${ }^{14}$, the current study shed light on the association of higher MES gene expression and low tumor purity, by identifying a 5ALA+ MES gene-signature which constitutes only a minor fraction of the invasive margin (we have previously shown that 5ALA+ cells constitute $\sim 5 \%$ of the Inv margin 633 $\left.{ }^{14}\right)$.

634

A recent study identified MTC and GPM metabolic states traits of GBM cells by 636 integrating multiple single cells and bulk tumor transcriptome datasets ${ }^{9}$. The authors concluded that a MTC state that relies on oxidative phosphorylation for energy production was associated with the most favorable clinical outcome, whilst a GPM state mediated by aerobic glycolysis and amino acid/lipid metabolism, was linked to poor patient outcome ${ }^{9}$. We provide evidence of GPM enrichment of GBM Core and Rim but not Inv. Interestingly, both GPM and MTC states were enriched in 5ALA+ and 5ALA-

642 cells, reflective of a shared microenvironment in controlling the metabolic states of both 643 infiltrative tumor and non-neoplastic cells.

644

645 Upregulation of exonic levels for MES and Inflammatory wound-response genes in $6465 A L A+$ relative to 5ALA- cells implied that these genes were under active post647 transcriptional control. Previously, a mass-spectrometry-based study has shown that 648 altered expression of nuclear regulatory proteins controlling transcription and post649 transcriptional processes may drive GBM invasion ${ }^{31}$. In addition to the differential 650 abundance of nuclear proteins, the role of the microenvironment in the regulation of 651 post-transcriptional processes including splicing and translational control cannot be 652 ruled out ${ }^{32}$. However, the unique genetic background and cell lineage of the 5ALA+ 
cells may contribute microenvironmental interaction distinct from that of normal neural cells residing in the infiltrative margin.

The transcriptional regulatory network that governs GBM transition to MES and recurrence has not been elucidated, partly due to an inability to identify and characterize rare GBM sub-populations exhibiting MES. The transition of GSCs to MES was reported to be dependent on TNF- $\alpha$ signaling via NFKB pathway ${ }^{13}$, which was upregulated in $5 A L A+$ cells in our study. Enrichment of MES both in the 5ALA+ subpopulation and recurrent tumors, offers a unique opportunity to explore 5ALA+ transcriptional networks further, to elucidate biomarkers predictive of recurrence interval and to identify putative molecular therapeutic targets to initiate treatment in advance of recurrence.

Interestingly, amongst the TFs, two NFKB family members - NFkB1 and REL - in addition to the inhibitor of NFKB -REL complex NFKBIA, were upregulated in 5ALA+ cells. These seemingly opposing factors may establish a delicate balance between inflammatory and anti-inflammatory pathways that are required to maintain a chronic and persistent low level of inflammation, further boosted by the infiltration of antiinflammatory and regulatory immune cells ${ }^{33}$. MES genes were mostly controlled by IRF8, NFkB1, and FOSL2. The upregulation of IRF8 in 5ALA+ cells was surprising, since it is considered a myeloid-lineage specific master TF exclusively restricted to the hematopoietic lineage ${ }^{34}$. Moreover, expression of IRF8 was assumed to be repressed in neural stem cells ${ }^{35}$ which raises the question of why and how 5ALA+ cells were able to express IRF8. In a recent study, Gangoso et al. shed light on this conundrum, by performing RNA-seq analysis of patient-derived GSC cultures, and based on differential immune-induced gene expression, identified two distinct subtypes - nonmesenchymal immune signature (Non-MESImm) and mesenchymal-immune signature (MESImm) ${ }^{35}$. Remarkably, an upregulation of interferon regulatory factor family members in MESImm-enriched GSCs (including IRF8) was observed, suggesting that GSCs have the capacity to hijack myeloid-specific transcriptional modules to evade the immune response and chemokine expression such as CCL2 ${ }^{35}$. As a mechanistic explanation of how MESImm GBM subtype cells are able to express IRF8 and CCL2, 
684 the authors observed hypomethylation at specific CpG islands associated with IRF8 and 685 CCL2 genes. These findings signify a possibility of epigenetic alteration reshaping the transcriptomics landscape of 5ALA+ cells to express IRF8 and CCL2. In agreement with 687 the current results, FOSL2 was previously confirmed as a contributing factor of MES 688 acquisition ${ }^{36}$.

689

The higher mRNAsi of 5ALA+ cells (which we have defined GBM Infiltrative Stem), relative to canonical stemness signatures of intra-tumor GBM regions and TCGA GBMs, urges pragmatic reflection on GSC biology to date. We reveal a higher mRNAsi in the GBM infiltrative margin relative to GBM Core and Rim, which may be explained by the presence of a higher number of neural progenitor cells in the GBM Inv region. Previously, we reported a distinct molecular composition of the heterogeneous GBM infiltrative margin characterized by higher stem cell marker expression compared to GBM Core ${ }^{2}$. The current study gained a deeper insight into the heterogeneity of the 698 GBM Inv by extending the hypothesis that molecularly distinct 5ALA+ cells exhibit the 699 highest stemness index. As non-neoplastic 5ALA- cells also exhibited a higher stemness profile, the microenvironment composing the GBM infiltrative margin is a 701 probable determinant in the acquisition of stemness. Miranda et al. investigated the link between stemness and immune cell infiltration across 21 cancer types including GBM 37, demonstrating a negative correlation between cancer stemness and anti-tumor 704 immune response as well as immunosuppressive pathways, independent of increased 705 706 mutation load, cancer-antigen expression and intratumoral heterogeneity ${ }^{37}$. Our observation of IRF8 and CCL2 upregulation in 5ALA+ cells may contribute to such immunosuppressive pathways by recruitment of regulatory immune cells.

708

709

Lastly, we associate 5ALA+ gene signatures with GBM recurrence. Gene-signatures representing stemness, MES and inflammatory wound response were enriched in recurrent GBM tumors relative to primary tumors and were associated with poor survival

712 outcome. Recently, Varn et al. showed that MES transition of GBM is linked to the 713 presence of a distinct myeloid cell state characterized by unique ligand-receptor 714 interactions with malignant cells ${ }^{12}$. This finding is consistent with our results showing 
715 MES subtype-enriched 5ALA+ cells may serve as the source of IRF8-mediated 716 signaling, leading to the production and secretion of chemokines such as CCL2, which 717 may in turn act as a chemo-attractive agent to recruit myeloid cells into the GBM 718 infiltrative margin.

Overall, our results comprehensively revealed unique molecular characteristics of the 5ALA+ sub-population(s) within the GBM infiltrative margin and underscored the possibility that interaction of 5ALA+ cells with this microenvironment may be a critical determinant for survival outcome and GBM recurrence. Our findings encourage the neuro-oncology research community to prioritize this infiltrative GBM subpopulation(s) for both mechanistic pre-clinical modeling and to expedite next-generation molecular targeted drug screening. Characterization of the 5ALA+ infiltrative sub-population offers an opportunity to develop more effective GBM treatments and urges focus away from the GBM proliferative core, upon which failed targeted therapies have been predicated.

\section{Methods}

Tissue sample collection, processing, and FACS analysis:

732 Tissue samples representing spatially distinct regions (Core, Rim, Inv) of tumor samples from 10 GBM patients were retrieved that were previously collected as part of an earlier study ${ }^{14}$. Consent and ethical approval was obtained from the Local Regional Ethics 735 Committee in Nottingham (East Midlands Ethics Approval Reference: 11/EM/0076). Briefly, 5ALA (20 mg/kg dose) was administered orally to patients $2-4 \mathrm{~h}$ prior to craniotomy and visualization of 5ALA induced fluorescence. Aided by image guidance, 738 multi-region tissue samples were collected from non-fluorescent or minimally fluorescent regions representing Core, whilst samples from the viable fluorescent region corresponded to Rim. The furthest region of 5ALA-induced fluorescence beyond the 741 bulk tumor, where the tumor penetrated adjacent healthy parenchyma, corresponded to 742 the Inv. Histological diagnosis and formal postoperative diagnosis (including IDH1 743 mutations, ATRX mutation, and MGMT methylation status) were included 744 (Supplementary Table S1). Cells were dissociated from the invasive margin and subjected to FACS based on 5ALA immunofluorescence as described by us previously 
14. Briefly, the cells were sorted using an excitation spectrum at $405 \mathrm{~nm}$ and an emission spectrum at 605-625 nm. The purity of the cells was ensured, by sorting the cells twice consecutively. As controls, U251 GBM cells incubated for 2 hours with and without 5ALA, were used for FACS gating. The positive and negative sorted cells were subsequently centrifuged at $800 \mathrm{rpm}(180 \times \mathrm{g})$ for 5 minutes before subjected to snap freezing.

\section{Immunohistochemistry}

Tissues from spatially distinct regions of GBM were collected and immunohistochemistry performed as described by us previously ${ }^{2}$. Samples were obtained from Core (superficial and anterior medial), Rim (deep edge), and Inv. Briefly, after the removal of paraffin wax, samples were treated with sodium citrate buffer $(\mathrm{pH} 6)$ for 40 minutes at $90^{\circ} \mathrm{C}$ and washed with phosphate buffer solution (PBS) for 2 minutes. Then, $200 \mu \mathrm{L}$ of peroxidase blocking solution was applied to cover the specimen for 5 minutes followed by washing with PBS. After the slides were dried, Ki-67 antibody (DAKO) and CD31 (DAKO) were applied at 1:50 dilution and incubated for 1 hour at room temperature. Sections were washed with PBS before the addition of the secondary antibody (DAKO) and incubated at $37^{\circ} \mathrm{C}$ for 30 minutes. Finally, substratechromogen solution (DAB) was applied to cover the specimen, incubated for 5 minutes, and rinsed gently with distilled water. An Olympus BX41 light microscope was used to visualize and capture the images of the each GBM region.

Immunohistochemistry for NeuN was performed on four patient tissue microarrays (TMAs) containing three intra-tumor regions of nine patient tumors in triplicate. The histology of the tissues was confirmed as GBM by an experienced pathologist. Following deparaffinisation through a xylene and alcohol series, antigens were retrieved via boiling sections in Tris-EDTA ( $\mathrm{pH}$ 9.0). Once cooled, sections were washed in PBS buffer, and then blocked using $20 \%$ normal goat serum. The sections were then incubated for 1 hour at room temperature with rabbit monoclonal neuronal marker NeuN (Abcam, ab177487) at a dilution of 1:3000. Following three washes with PBS the sections were incubated with secondary antibody (Dako Chemate EnVision kit) for 1 
777 hour at room temperature. Sections were then incubated with DAB-chromogen complex 778 and incubated for 5 minutes. Counterstaining was performed with haematoxylin before rehydrating the sections by passing through the previous alcohol to xylene series. Sections were scored and neuron numbers counted, and statistical analysis via student t-test performed using Graphpad Prism (9.0).

\section{RNA isolation and RNA-seq library preparation}

Dissociation of Core, Rim and Inv was performed as previously described by us ${ }^{38}$. Total RNA extraction followed by quality control analysis was performed as described by us ${ }^{14}$. Briefly, libraries were prepared using the NEBNext Poly $(A)$ mRNA Magnetic Isolation Module (NEB: E7490), the NEBNext Ultra Directional Library Kit for Illumina (NEB: E7420) and the NEBNext Multiplex Oligos for Illumina (Index Primers Set 1) (NEB: E7335L). Samples with total RNA concentration of $>10 \mathrm{ng} / \mu \mathrm{l}(0.5 \mu \mathrm{g}$ total amount) were used for library preparation. To ensure library quality, adequate concentrations were obtained from each sample, followed by 14 cycles of amplification during the PCRbased library enrichment step. Finished libraries were quantified using the Qubit dsDNA HS kit (Invitrogen: Q32854). Library concentrations as well as fragment size distributions were also analyzed by employing the Agilent Bioanalyzer High Sensitivity DNA Kit (Agilent: 5067-4626). Libraries were normalized to $2 \mathrm{nM}$ and pooled in equimolar amounts. The Kapa Library Quantification Kit (KAPA Biosystems: KK4824) was used for precise quantification of the library pool. The library pool was denatured and diluted to $1.6 \mathrm{pM}$, spiked with $1 \%$ PhiX (1.8 pM) and sequenced on the Illumina NextSeq 500, using the NextSeq 500/550 High Output v2 Kit (150 cycles) (Illumina: FC404-2005), to generate a minimum of 70 million pairs of 75-bp paired-end reads per sample. Raw RNA-seq data has been deposited at ArrayExpress with accession number E-MTAB-8743.

\section{RNA-seq analysis}

We obtained RNA-seq raw data (FASTQ files) from spatially distinct unsorted regions (Core, Rim, and Inv) and 5ALA sorted cells from the Inv across 10 GBM patients. RNAseq raw data from spatially distinct regions were processed using Bioconductor 
package QuasR (version 1.30.0) ${ }^{39}$. For primary alignment, we used the reference genome hg19 for human. The QuasR package employs the required tools to obtain expression tables from the raw RNA-seq reads and includes the aligners Rhisat ${ }^{40}$ and SpliceMap ${ }^{41}$. We performed the alignment by using the following command: 'qAlign(“sampleFile.txt”,"BSgenome.Hsapiens.UCSC.hg19",splicedAlignment=TRUE, aligner="Rhisat2")'.

We then measured the count of each gene within any annotated exonic region using the function qCount. The count data obtained from the QuasR package was then converted to transcripts per million (TMP) followed by log transformation $\left(\log _{2}\right)$ of $T P M+1$ values. Initial quality control analysis was performed on the normalized RNA-seq counts including a comparison of differential expression across the samples. Genes with valid count values were additionally compared amongst the samples.

\section{Hallmark gene-set enrichment analysis (GSEA)}

Enrichment analyses of the hallmark gene sets representing biological processes related to cancer were carried out by a GSEA algorithm ${ }^{42}$. Briefly, the hallmark gene sets were selected from MSigDB gene-set collections ${ }^{43}$, and enrichment analysis was conducted amongst the different regions (Core, Rim, and Inv) and 5ALA sorted cells (5ALA+ and 5ALA- cells) using GSEA. The ranked list of genes obtained from GSEA was further processed by Fast Gene Set Enrichment Analysis (fgsea) R-package ${ }^{44}$. Normalized enrichment score (NES), p-value, and adjusted p-values (calculated with a standard Benjamini-Hochberg - $\mathrm{BH}$ procedure) were retrieved for each of the hallmarks that were enriched in different regions and cell populations. The hallmarks with higher NES values and adjusted $p$-value $<0.05$ were considered as enriched for a specific GBM region. For further analysis of the enriched pathways, the leading edge genes representing the subset of genes contributing significantly to the enrichment signal of a given gene set ${ }^{42}$ in a specific GBM region, were identified and subjected to hierarchical clustering analysis. We employed a combined ComplexHeatmap::pheatmap() function that uses two R-packages, 'pheatmap' and 'ComplexHeatmap', where the column and row clustering were performed by using the Euclidean distance method. 
839 Neural cell-type gene-signature enrichment

840 To characterize the different GBM regions, transcriptome-based neural cell-type 841 signatures described by Cahoy et al. were retrieved ${ }^{45}$ (Supplementary Table S1). In 842 brief, Cahoy et al. employed Affymetrix GeneChip Arrays to identify gene-signatures of 843 different neural cell types including neurons, oligodendrocytes, astrocytes, and cultured 844 astroglial cells ${ }^{45}$. NES and adjusted $p$-values were calculated using GSEA and fgsea 845 algorithms as described above.

\section{GBM subtype gene-signature enrichment}

848 Gene signatures of each GBM molecular subtype were obtained from Verhaak et al. 849 which described an efficient gene expression-based molecular classification of GBM 850 samples into four molecular subtypes: PN, NE, CL and MES ${ }^{10}$ (Supplementary Table 851 S1). The signature gene-set for each of the subtypes was retrieved from MSigDB. 852 GSEA for the molecular subtypes was performed on the spatially distinct GBM regions.

853 NES and adjusted p-values were calculated using the fgesa package as described 854 previously.

Developmental, inflammatory wound response, and metabolic gene signature enrichment

858 Signature genes for developmental and inflammatory wound healing/ injury response 859 phenotypes were retrieved from Richards et al. ${ }^{8}$ (Supplementary Table S1). In addition, 860 two gene-sets representing two divergent metabolic phenotypes - mitochondrial (MTC), 861 glycolytic/plurimetabolic (GPM) were retrieved from Garofano et al. ${ }^{9}$ 862 (SupplementaryTable S1). GSEA was performed to identify the enrichment of these 863 diverse gene-sets in spatially distinct GBM regions.

864

\section{Stemness gene-signature enrichment}

Diverse signature gene-sets representing stem cells ${ }^{23}$, cancer stemness ${ }^{37}$, embryonic stem cells (ES1 and ES2) ${ }^{25}$, Human Embryonic Stem Cells (hESC) ${ }^{22}$, induced

868 pluripotent stem cells (iPSC) ${ }^{21}$, Nonog/Sox2 induced stem cell gene-set ${ }^{25}$, Myc 869 induced ES gene-set ${ }^{24}$ and Human Epithelial adult stem cells ${ }^{46}$ were retrieved 
870 (Supplementary Table S1). GSEA was performed to identify the enrichment of these 871 diverse gene-sets in spatially distinct GBM regions as described earlier.

872

873

874

875

876

877

878

879

880

881

882

883

884

885

886

887

888

889

890

891

892

893

894

895

896

897

898

899

900

\section{Estimation of mRNA expression-based stemness index (mRNAsi)}

Estimation of mRNA expression-based stemness index (mRNAsi) was performed by the method described by Malta et al. ${ }^{19}$. Briefly, to calculate mRNAsi, a machine learning approach was used to develop a predictive model by employing one-class logistic regression (OCLR) as described previously by Sokolov et al. ${ }^{47}$. The OCLR was based on the hESC and iPSC from the Progenitor Cell Biology Consortium (PCBC) dataset ${ }^{48}$, 49 . The mRNAsi score ranges between 0 and 1 , where 0 indicates less stemness with a more differentiated tissue state and 1 represents more stemness with a less differentiated state. To generate a stemness score based on the spatial RNA-seq data from 10 patients, and in comparison to The Cancer Genome Atlas (TCGA) GBM samples, a gene expression matrix (samples in columns and genes in rows) was prepared and employed on R-package "TCGAbiolinks" using the function TCGAanalyze_Stemness().

\section{Construction of the transcriptional network}

Firstly, transcription factors in the leading gene sets representing cellular and metabolic states including inflammatory pathway, tumor necrosis factor- $\alpha$ (TNF- $\alpha$ ) signaling via nuclear factor-кB (NFKB) pathway, MES subtype, inflammatory wound response, MTC, and GPM subtypes, were manually curated using the ENCODE database ${ }^{50}$. We used the mutual information-based algorithm ARACNE ${ }^{51}$ to construct the regulatory network between transcription factors and target genes based on mRNA expression values of the leading edge genes. The previously described bootstrap algorithm ${ }^{52}$ was used to assess statistical confidence. We inferred 1000 networks based on bootstrap datasets, setting the most stringent value for Data Processing Inequality $(\mathrm{DPI}=0)$ tolerance. Finally, we estimated the significance of the edges by testing their probability against a null distribution obtained by random permutation of predicted edges. The consensus network conserves edges with a $p$-value $<10^{-4}$. Based on the ARACNE output we retrieved only the mutual information (MI) values for a given 
901 transcription factor and target genes for which the $p$-value was significant $(<0.05)$, and 902 constructed and visualized the transcription factor - target genes network using 903 Cytoscape ${ }^{53}$.

904

905

906

907

908

909

910

911

912

913

914

915

916

917

918

919

920

921

922

923

924

925

926

927

928

929

930

931

\section{Survival analysis}

The hazard ratios for individual genes were estimated by Cox proportional hazards model regression analysis ${ }^{54}$ based on TCGA GBM cohort (https://tcgadata.nci.nih.gov/tcga/). Analysis was performed at a $95 \%$ confidence interval. To investigate the impact of gene-signatures on survival outcome, a Multivariate Cox analysis based on TCGA mRNA $\log _{2}$ expression data $(T P M+1)$ of gene signatures was performed using GEPIA2 ${ }^{55}$. Briefly, the survival curves were generated using KaplanMeier analysis for overall and disease-free survival by using a median survival cutoff. Survival analysis for each of the gene signatures representing Inflammatory response, TNF- $\alpha$ signaling, MES subtype, Inflammatory wound response, and metabolic subtypes (GPM and MTC) was performed separately. For classifying the high- and lowexpression cohorts, the median expression threshold was set (cut-off high: upper $50 \%$ and cut-off low: lower $50 \%$ ).

\section{Exon-intron split analysis (EISA)}

Exon-intron split analysis (EISA) was employed as described by Gaidatzis et al. ${ }^{18}$ to investigate the changes in pre-mRNA (introns) and mature-mRNA (exons) counts across different regions of GBM, that leads to the quantification of transcriptional and post-transcriptional control of gene expression. R-package "eisaR" was used for the EISA. Briefly, after mapping the transcripts to a unique position in the genome, counts of annotated exonic reads representing mature mRNAs were quantified, in addition to read counts that did not match any annotated exons (intronic). Normalization was performed for exons and introns separately by dividing each sample by the total number of reads and multiplying by the average library size. Based on these expression levels, only the genes with reasonable counts (average $\log _{2}$ expression level of at least 5 ) were selected for downstream analysis. Genes with overlapping reads were discarded due to difficulty assigning intronic reads to the respective genes. A differential exonic and 
932 intronic change among different regions was been performed with EdgeR, as described 933 by Gaidatzis et al. where a p-value < 0.05 was considered significant. GSEA of 934 Hallmark, 5ALA+ cell-derived signatures, and transcription factors were conducted as 935 described before, with genes ranked based on the $\log _{2}$ difference of exon or intron 936 normalized intensity between two regions used as input.

\section{Recurrent vs. Primary GBM analysis}

939 To compare the gene-signature enrichments in IDH wild-type recurrent and primary GBM tumors, RNA-seq data was retrieved from TCGA ( $N=140$; Primary = 128 and Recurrent = 12) (https://tcga-data.nci.nih.gov/tcga/), Chinese Glioma Genome Atlas (CCGA) (N = 190; Primary = 109 and Recurrent = 81) (http://www.cgga.org.cn/) ${ }^{56}$ and 943 The Glioma Longitudinal AnalySiS (GLASS) $(\mathrm{N}=60$; Primary $=30$ and Recurrent $=30$ ) $944{ }^{57}$ cohorts. Gene signatures comprised of the leading edge genes representing TNF- $\alpha$ 945 signaling, inflammatory response, MES subtype, inflammatory wound response, MTC 946 subtype, GPM subtype, stemness and transcription factors that were upregulated in $5 A L A+$ cells, were used for enrichment. GSEA was performed to identify the enrichment of these diverse gene sets in spatially distinct GBM regions as described earlier. In order to investigate the correlation between the unique 5ALA+ gene-signatures and survival of GBM patients, a single sample GSEA ${ }^{58}$ was performed to identify the enrichment of Mesenchymal and Wound Response pathways for each primary and recurrent tumor samples from TCGA, CCGA and GLASS cohorts. NES for each of the gene sets were accumulated to calculate the 5ALA+ gene-signature score for each

954 primary and recurrent GBM patient. The Spearman correlation coefficient was 955 calculated between the 5ALA+ gene-signature scores of GBM patients and survival data 956 for primary and recurrent GBM separately. 
964 1. Stupp R, et al. Radiotherapy plus concomitant and adjuvant temozolomide for glioblastoma. The New England journal of medicine 352, 987-996 (2005).

2. Smith SJ, Diksin M, Chhaya S, Sairam S, Estevez-Cebrero MA, Rahman R. The Invasive Region of Glioblastoma Defined by 5ALA Guided Surgery Has an Altered Cancer Stem Cell Marker Profile Compared to Central Tumour. International journal of molecular sciences 18, (2017).

3. Lee $\mathrm{JK}$, et al. Spatiotemporal genomic architecture informs precision oncology in glioblastoma. Nature genetics 49, 594-599 (2017).

4. Sottoriva A, et al. Intratumor heterogeneity in human glioblastoma reflects cancer evolutionary dynamics. Proceedings of the National Academy of Sciences of the United States of America 110, 4009-4014 (2013).

5. Lan $\mathrm{X}$, et al. Fate mapping of human glioblastoma reveals an invariant stem cell hierarchy. Nature 549, 227-232 (2017).

6. Singh SK, et al. Identification of human brain tumour initiating cells. Nature $\mathbf{4 3 2}$, 396-401 (2004).

7. Chen J, et al. A restricted cell population propagates glioblastoma growth after chemotherapy. Nature 488, 522-526 (2012).

8. Richards LM, et al. Gradient of Developmental and Injury Response transcriptional states defines functional vulnerabilities underpinning glioblastoma heterogeneity. Nature Cancer 2, 157-173 (2021).

9. Garofano L, et al. Pathway-based classification of glioblastoma uncovers a mitochondrial subtype with therapeutic vulnerabilities. Nature Cancer 2, 141-156 (2021).

10. Verhaak $R G$, et al. Integrated genomic analysis identifies clinically relevant subtypes of glioblastoma characterized by abnormalities in PDGFRA, IDH1, EGFR, and NF1. Cancer cell 17, 98-110 (2010).

11. Wang Q, et al. Tumor Evolution of Glioma-Intrinsic Gene Expression Subtypes Associates with Immunological Changes in the Microenvironment. Cancer cell 32, $42-56$ e46 (2017). 
993 12. Varn FS, et al. Longitudinal analysis of diffuse glioma reveals cell state dynamics at recurrence associated with changes in genetics and the microenvironment.

14. Smith SJ, et al. Metabolism-based isolation of invasive glioblastoma cells with

15. Petrecca K, Guiot MC, Panet-Raymond V, Souhami L. Failure pattern following (2021).

13. Minata M, et al. Phenotypic Plasticity of Invasive Edge Glioma Stem-like Cells in Response to lonizing Radiation. Cell reports 26, 1893-1905 e1897 (2019). specific gene signatures and tumorigenic potential. Neuro-oncology advances $\mathbf{2}$, vdaa087 (2020). complete resection plus radiotherapy and temozolomide is at the resection margin in patients with glioblastoma. Journal of neuro-oncology 111, 19-23 (2013).

16. Stummer W, et al. Fluorescence-guided surgery with 5-aminolevulinic acid for resection of malignant glioma: a randomised controlled multicentre phase III trial. The Lancet Oncology 7, 392-401 (2006).

17. Lau D, et al. A prospective Phase II clinical trial of 5-aminolevulinic acid to assess the correlation of intraoperative fluorescence intensity and degree of histologic cellularity during resection of high-grade gliomas. Journal of neurosurgery 124, 1300-1309 (2016).

18. Gaidatzis D, Burger L, Florescu M, Stadler MB. Analysis of intronic and exonic reads in RNA-seq data characterizes transcriptional and post-transcriptional regulation. Nature biotechnology 33, 722-729 (2015).

19. Malta TM, et al. Machine Learning Identifies Stemness Features Associated with Oncogenic Dedifferentiation. Cell 173, 338-354 e315 (2018).

20. Zhang X, et al. CellMarker: a manually curated resource of cell markers in human and mouse. Nucleic acids research 47, D721-D728 (2019).

21. Shats I, et al. Using a stem cell-based signature to guide therapeutic selection in cancer. Cancer research 71, 1772-1780 (2011).

22. Bhattacharya $B$, et al. Gene expression in human embryonic stem cell lines: unique molecular signature. Blood 103, 2956-2964 (2004). 
1023 23. Palmer NP, Schmid PR, Berger B, Kohane IS. A gene expression profile of stem

1024

1025

1026

1027

1028

1029

1030

1031

1032

1033

1034

1035

1036

1037

1038

1039

1040

1041

1042

1043

1044

1045

1046

1047

1048

1049

1050

1051

1052

1053 cell pluripotentiality and differentiation is conserved across diverse solid and hematopoietic cancers. Genome biology 13, R71 (2012).

24. Kim J, et al. A Myc network accounts for similarities between embryonic stem and cancer cell transcription programs. Cell 143, 313-324 (2010).

25. Ben-Porath I, et al. An embryonic stem cell-like gene expression signature in poorly differentiated aggressive human tumors. Nature genetics 40, 499-507 (2008).

26. Gill BJ, et al. MRI-localized biopsies reveal subtype-specific differences in molecular and cellular composition at the margins of glioblastoma. Proceedings of the National Academy of Sciences of the United States of America 111, 12550-12555 (2014).

27. Almiron Bonnin DA, et al. Characterizing the heterogeneity in 5-aminolevulinic acid-induced fluorescence in glioblastoma. Journal of neurosurgery 132, 17061714 (2019).

28. Martinez E, Yoshihara K, Kim H, Mills GM, Trevino V, Verhaak RG. Comparison of gene expression patterns across 12 tumor types identifies a cancer supercluster characterized by TP53 mutations and cell cycle defects. Oncogene 34, 2732-2740 (2015).

29. Yoshihara $\mathrm{K}$, et al. Inferring tumour purity and stromal and immune cell admixture from expression data. Nature communications 4, 2612 (2013).

30. Behnan J, Finocchiaro G, Hanna G. The landscape of the mesenchymal signature in brain tumours. Brain : a journal of neurology 142, 847-866 (2019).

31. Gupta MK, et al. Altered transcriptional regulatory proteins in glioblastoma and YBX1 as a potential regulator of tumor invasion. Scientific reports 9, 10986 (2019).

32. Jewer M, Findlay SD, Postovit LM. Post-transcriptional regulation in cancer progression : Microenvironmental control of alternative splicing and translation. Journal of cell communication and signaling 6, 233-248 (2012).

33. Grivennikov SI, Greten FR, Karin M. Immunity, inflammation, and cancer. Cell 140, 883-899 (2010). 
1054 34. Driggers $\mathrm{PH}$, et al. An interferon gamma-regulated protein that binds the 1055

35. Gangoso E, et al. Glioblastomas acquire myeloid-affiliated transcriptional 1059

36. Carro MS, et al. The transcriptional network for mesenchymal transformation of 1062

37. Miranda A, et al. Cancer stemness, intratumoral heterogeneity, and immune response across cancers. Proceedings of the National Academy of Sciences of the United States of America 116, 9020-9029 (2019).

38. Hussein D, et al. Pediatric brain tumor cancer stem cells: cell cycle dynamics, DNA repair, and etoposide extrusion. Neuro-oncology 13, 70-83 (2011).

39. Harvey CT, Moyerbrailean GA, Davis GO, Wen X, Luca F, Pique-Regi R. QuASAR: quantitative allele-specific analysis of reads. Bioinformatics 31, 12351242 (2015).

40. Kim D, Langmead B, Salzberg SL. HISAT: a fast spliced aligner with low memory requirements. Nature methods 12, 357-360 (2015).

41. Au KF, Jiang $H$, Lin $L$, Xing $Y$, Wong $W H$. Detection of splice junctions from paired-end RNA-seq data by SpliceMap. Nucleic acids research 38, 4570-4578 (2010).

42. Subramanian A, et al. Gene set enrichment analysis: a knowledge-based approach for interpreting genome-wide expression profiles. Proceedings of the National Academy of Sciences of the United States of America 102, 1554515550 (2005).

43. Liberzon A, Birger C, Thorvaldsdottir H, Ghandi M, Mesirov JP, Tamayo P. The Molecular Signatures Database (MSigDB) hallmark gene set collection. Cell systems 1, 417-425 (2015).

44. Sergushichev A, A. An algorithm for fast preranked gene set enrichment analysis using cumulative statistic calculation. bioRxiv preprint, (2016). 
1085

1086

1087

1088

1089

1090

1091

1092

1093

1094

1095

1096

1097

1098

1099

1100

1101

1102

1103

1104

1105

1106

1107

1108

1109

1110

1111

1112

1113

1114

45. Cahoy JD, et al. A transcriptome database for astrocytes, neurons, and oligodendrocytes: a new resource for understanding brain development and function. The Journal of neuroscience : the official journal of the Society for Neuroscience 28, 264-278 (2008).

46. Smith BA, et al. A Human Adult Stem Cell Signature Marks Aggressive Variants across Epithelial Cancers. Cell reports 24, 3353-3366 e3355 (2018).

47. Sokolov A, Paull EO, Stuart JM. One-Class Detection of Cell States in Tumor Subtypes. Pacific Symposium on Biocomputing Pacific Symposium on Biocomputing 21, 405-416 (2016).

48. Daily $\mathrm{K}$, et al. Molecular, phenotypic, and sample-associated data to describe pluripotent stem cell lines and derivatives. Scientific data 4, 170030 (2017).

49. Salomonis N, et al. Integrated Genomic Analysis of Diverse Induced Pluripotent Stem Cells from the Progenitor Cell Biology Consortium. Stem cell reports 7, 110-125 (2016).

50. Consortium EP. A user's guide to the encyclopedia of DNA elements (ENCODE). PLoS biology 9, e1001046 (2011).

51. Margolin AA, et al. ARACNE: an algorithm for the reconstruction of gene regulatory networks in a mammalian cellular context. BMC bioinformatics 7 Suppl 1, S7 (2006).

52. Margolin AA, Wang K, Lim WK, Kustagi M, Nemenman I, Califano A. Reverse engineering cellular networks. Nature protocols 1, 662-671 (2006).

53. Kohl M, Wiese S, Warscheid B. Cytoscape: software for visualization and analysis of biological networks. Methods in molecular biology 696, 291-303 (2011).

54. Moolgavkar SH, Chang ET, Watson HN, Lau EC. An Assessment of the Cox Proportional Hazards Regression Model for Epidemiologic Studies. Risk analysis : an official publication of the Society for Risk Analysis 38, 777-794 (2018).

55. Tang Z, Kang B, Li C, Chen T, Zhang Z. GEPIA2: an enhanced web server for large-scale expression profiling and interactive analysis. Nucleic acids research 47, W556-W560 (2019). 
1115 56. Zhao Z, et al. Chinese Glioma Genome Atlas (CGGA): A Comprehensive 1116 Resource with Functional Genomic Data from Chinese Gliomas. Genomics, 1117 proteomics \& bioinformatics, (2021).

1118 57. Consortium G. Glioma through the looking GLASS: molecular evolution of diffuse 1119 gliomas and the Glioma Longitudinal Analysis Consortium. Neuro-oncology 20, 1120 873-884 (2018).

1121 58. Barbie DA, et al. Systematic RNA interference reveals that oncogenic KRAS1122 driven cancers require TBK1. Nature 462, 108-112 (2009).

1123

1124

1125

1126

1127

1128

1129

1130

1131

1132

1133

1134

1135

1136

1137

1138

1139

1140

1141

1142

1143

1144

1145

1146

1147

1148

1149

1150

1151

1152

1153

1154

1155

1156 
1157 Data availability:

1158

1159

1160

1161

1162

1163

1164

1165

1166

1167

1168

1169

1170

1171

1172

1173

1174

1175

1176

1177

1178

1179

1180

1181

1182

1183

1184

1185

1186

1187

1188

1189

Raw RNA-seq data for spatially distinct unsorted GBM regions and 5ALA FACS sorted cells have been deposited at the ArrayExpress with accession number E-MTAB-8743. The full datasets used in the current paper are available at https://github.com/dtonmoy/GBM-project.

\section{Code availability}

All the codes (R-scripts) used to analyze the data and visualization of the results are available through https://github.com/dtonmoy/GBM-project.

\section{Acknowledgements}

The study was funded by a Brain Tumour Charity grant (REF: GN-000345) awarded to SJS and RR. Additional funding to MB by the Deutsche Forschungsgemeinschaft (DFG, German Research Foundation) - SFB 850 subprojects C9 and Z1 and SFB1479 Project ID: 441891347- S1, SFB1160, SFB1453, TRR167, the German Federal Ministry of Education and Research by MIRACUM within the Medical Informatics Funding Scheme (FKZ 01ZZ1801B).

\section{Author contributions}

GA, TD, MG, MB, SJS, RR, and SC conceptualized the manuscript. SJS performed the 5ALA fluorescence guided neurosurgery and collected spatially distinct tumor regions. SJS and RR conducted the 5ALA fluoresce based FACS sorting and generated the spatial transcriptomics (RNA-seq) data. MG performed the immunohistochemistry experiments. GA, TD, and $S C$ analyzed data and prepared the figures. GA, TD, MG, $\mathrm{MB}, \mathrm{SJS}, \mathrm{RR}$, and SC wrote and approved the manuscript.

\section{Competing interests}

The authors declare no competing interests. 
Figure 1: Hallmark gene set enrichment analysis (GSEA) in spatially distinct different GBM.

1194 Schematic figure delineating the steps for tissue collection from distinct GBM regions ( Core, Rim and Inv margin) followed by 5ALA fluorescence-based FACS isolation of Inv cells into 5ALA+ and 5ALA- subpopulations. The spatial-transcriptome from each unsorted region and sorted cells were interrogated by gene-set enrichment analysis (GSEA), hierarchical clustering, differential expression analysis, Exon-intron split analysis (EISA), network inference and one class logistic regression (OLCR) (A). The hallmark gene sets were obtained from the MSigDB database followed by GSEA based on the mRNA expression dataset from different GBM regions. The heatmap represents the normalized enrichment scores (NES) representing the degree to which a particular hallmark is significantly enriched (padj<0.05) in a specific GBM region. The color represents the value of NES where yellow and black indicate the highest $(\mathrm{NES}=3.5)$ and lowest (NES = 0) NES values, respectively $(B)$. Ki67 immunohistochemistry (IHC) of tumor core - superficial medial (C), anterior medial (D), tumor rim (E) and invasive margin $(F)$ to estimate the fraction of proliferating cells in spatially distinct regions of GBM. The scale bar indicates $25 \mu \mathrm{m}$. CD31-IHC exploring tumor vascularity identifying the number of vascular structure in tumor core $(\mathrm{G})$, tumor rim $(\mathrm{H})$ and invasive margin (I). The scale bar indicates $100 \mu \mathrm{m}$. Heatmaps showing hierarchical clustering based on z-scored expression $\left(\log _{2} \mathrm{TPM}+1\right)$ of the leading edge genes of selected hallmarks -

1212 hypoxia $(\mathrm{J})$, and inflammatory response/ TNF- $\alpha$ signaling via NFKB (K). The leading 1213 edge genes representing the genes that contribute significantly to higher NES were 1214 identified from the GSEA output. Both the rows (genes) and columns (samples) were 1215 clustered using the Euclidian distance method. The column samples are further 1216 separated into different regions: Core, Rim, Inv, 5ALA sorted cells (5ALA+ and 5ALA-). 1217 The color code indicates the differential expression of leading-edge genes where red 1218 and blue represent higher and lower expressions, respectively. Some rows are labeled 1219 with the symbols of selected genes of interest. 
1221 Figure 2: Enrichment of GBM subtypes in distinct GBM regions.

1222 Retrieval of gene-sets specifying different GBM subtypes was followed by GSEA. The 1223 NES of the significantly enriched (padj<0.05) GBM subtypes are shown in distinct GBM 1224 regions (Core, Rim, Inv) and 5ALA sorted cells (5ALA- and 5ALA+) $(A)$. The color code 1225 indicates the differential NES values where yellow and black represent higher and lower 1226 normalized enrichment scores (NES) respectively. GSEA plot shows that the GBM 1227 mesenchymal subtype-specific genes are significantly enriched (NES: 2.1, padj= $\left.12282.3 \times 10^{-6}\right)$ in $5 A L A+$ cells $(B)$. NeuN immunohistochemistry (IHC) of Core - (C), Rim (D) 1229 and Inv (E) to estimate the proportion of NeuN positive (neuronal) cells in spatially 1230 distinct regions of GBM. The scale bar indicates $25 \mu \mathrm{m}$. Arrows indicate the NeuN 1231 positive cells. Differential z-scored normalized expression $\left(\log _{2} T P M+1\right)$ of the leading 1232 edge genes of GBM subtypes - Classical (F), Neural $(G)$, Proneural $(H)$, and 1233 Mesenchymal (I) - are shown as a heatmap. Column (samples) and row (genes) are 1234 clustered by the Euclidian distance method. The color code represents the differential 1235 expression of genes (Yellow: higher expression; Blue = lower expression).

Figure 3: Enrichment of cellular and metabolic subtypes in distinct GBM regions.

1238 The gene-signatures of cellular states (Developmental and Inflammatory wound 1239 response) and metabolic states (Glycolytic - GPM and Mitochondrial - MTC) were 1240 retrieved and subjected to GSEA. Heatmap illustrating the normalized enrichment 1241 scores (NES) representing enriched cellular and metabolic states with (padj<0.05) in 1242 distinct GBM intra-tumor regions and 5ALA sorted cells (A). GSEA plots representing 1243 the enrichment of GPM-associated genes (B), MTC (C), and Inflammatory wound 1244 response (D) enriched in 5ALA+ cells compared to 5ALA- cells, Rim and Inv 1245 respectively. Heatmaps showing the differential expression of leading-edge genes of 1246 GPM (E), MTC (F), and Inflammatory wound response $(G)$ in Core, Rim, Inv, 5ALA- and 12475 SLA+ cells. [Inf. wound response $=$ Inflammatory wound response]. Figure 4: Construction of transcription networks upregulated in 5ALA+ cells

1250 Transcription factors (TF) were identified in the leading edge genes that were enriched 1251 in 5ALA+ cells by using ENCODE TF-annotation. The association between TF and 
1252 corresponding target genes (TG) was retrieved from the ENCODE database. A 1253 transcription network is constructed representing TF-TG association by using the 1254 ARACNe algorithm. The diagram illustrates that TGs (Nodes) associated with enriched 1255 gene sets (Inflammatory response, Inflammatory wound response, MTC, Mesenchymal 1256 subtype, GPM and TNFA signaling), are mutually controlled by different TFs (Hubs). 1257 The association between a node (TG) and a hub (TF) is shown as edges (grey curved 1258 lines) and nodes/hubs are color-coded according to the $\log _{2}$ fold change (FC) values 1259 between 5ALA+ and 5ALA- cells (A), where red and blue indicate higher and lower FCs 1260 respectively. A radar plot representing TG (of different enriched subtypes) counts of the 1261 eight TFs (B). The colored dots represent the TG count for a specific TF.

Figure 5: Exon-intron split analysis (EISA) of enriched cellular and metabolic subtypes in 5ALA+ cells

Scatter plot showing the gene-specific changes in exon (delta-exon) and intron (deltacells (D). X- and $\mathrm{Y}$-axis represent the delta-intron and delta-exon, respectively. Color code represents the genes that showed significant regulation with adjusted $p$ value $<0.05$ (Blue: significant change in delta intron and Red: significant change in delta exon) between 5ALA+ cells and intra-tumor regions or 5ALA- cells. Heatmap showing 1271 the -log10 adjusted p-value of the hallmark enriched pathways (E). The samples representing the comparisons of $5 \mathrm{ALA}+$ with other regions are given in columns (5ALA+ vs. tumor Core; 5ALA+ vs. Rim; 5ALA+ vs. Inv ; and 5ALA+ vs. 5ALA-). Each row 1274 represents the different cellular and metabolic subtypes. Columns are divided into 1275 upregulated (Red) or downregulated (Blue) segments based on the exon and intron 1276 regulation of genes in 5ALA+ cells. Finally, delta-exon (yellow) and delta-intron (Light 1277 blue) are annotated for each column. The color code in the heatmap is representative of -log10 p-values (Orange is highly significant and Black is not significant). mRNA-based stemness index (mRNAsi) values across distinct GBM regions (Core, 
1283 are scaled between 0 (low) and 1 (high) (A) and are indicated by the gradient color 1284 code. Row (samples) and columns (GBM regions) are clustered by using a correlation 1285 algorithm. Comparison of the mRNAsi values according to brain regions (left) and 1286 TCGA-GBM samples (right) are shown as bar diagrams (B). The TCGA-GBM samples 1287 were pre-stratified according to GBM subtypes as described by Verhaak et al. ${ }^{10}$ 1288 Kruskal-wallis tests showed a significantly higher mRNAsi in 5ALA+ cells compared to 1289 Core and Rim, with $p$-values shown. Correlation (Pearson correlation coefficient) values 1290 between the mRNAsi and mRNA expression of selected genes are shown (C). The 1291 genes that showed a significant ( $p$-value $<0.05$ ) positive correlation with mRNAsi for 1292 5ALA+ cells for each patient were selected. Correlation values are indicated by the color 1293 code where red and blue represent positive and negative correlation respectively. The 1294 scatter plots show the correlation of mRNAsi values and mRNA expression of three 1295 selected genes (IL6, ABCA13, and FHL2) (D). Hazard ratio (HR) of the three genes 1296 IL6, $A B C A 13$, and FHL2 with confidence interval and p-values are shown (E).

Figure 7: Association of 5ALA+ gene-signatures with overall survival and recurrence in GBM patients.

1300 Based on the median combined mRNA expression of five different cellular and 1301 metabolic subtypes (TNF- $\alpha$ signaling, Inflammatory response, Mesenchymal subtype, Inflammatory wound response and GPM), TCGA-GBM patients were stratified into high-

$1303(\mathrm{~N}=81)$ and low-expression $(\mathrm{N}=81)$ groups. Only the genes that showed a significant 1304 association with GBM (hazard ratio>1 and $p$-value $<0.05$ ) in univariate analysis were 1305 considered for each subtype. The number of genes in each subtype is shown: TNF- $\alpha$ signaling $(N=27)$, Inflammatory response $(N=23)$, Mesenchymal subtype $(N=42)$, Inflammatory wound response $(\mathrm{N}=30)$ and GPM $(\mathrm{N}=11)$. Log-rank $p$-value indicates the significant difference in survival outcomes between high- and low-expression cohorts. Kaplan-Meier curves represent the overall survival outcomes of TNF- $\alpha$ signaling (A), Inflammatory response (B), Mesenchymal (C) Inflammatory wound response (D) and GPM (E) gene signatures respectively. Heatmap showing the -log10 1312 adjusted $p$-value of the enriched gene signatures across recurrent and primary GBM 1313 tumors $(F)$. The conditions representing the comparisons between recurrent and 
1314 primary GBM tumors from TCGA and CCGA are given in columns (TCGA - Recurrent 1315 vs. Primary and CCGA - Recurrent vs. Primary). GBM data from TCGA has been 1316 analyzed in a paired and unpaired manner. Columns are divided into upregulated (Red) 1317 or downregulated (Blue) segments based on the regulation of genes between Recurrent 1318 vs. Primary samples. Each row represents the different gene signatures. The color code 1319 in the heatmap is representative of $-\log 10$ p-values (Orange is high and Black is low). 1320 Scatter plots representing the correlation between 5ALA+ gene-signature scores 1321 (combined normalized enrichment score for Mesenchymal and Inflammatory wound 1322 response for each patient) and overall survival (months) in recurrent and primary 1323 patients from TCGA ( $G$ and $H$ ) and GLASS ( $I$ and J) cohorts. Spearman correlation 1324 coefficient $(R)$ and $p$-values are shown.

\section{Supplementary Figure S1}

1327 The boxplot of log normalized TPM expression values of 50 samples from 10 patients 1328 divided into five brain regions (Core, Rim, Inv, 5ALA-, 5ALA+) (A). The scatter plot 1329 matrix shows the histogram and correlation coefficients of all related brain regions. The 1330 lower triangle boxes represent the pairwise scatter plots, diagonal boxes show the brain 1331 regions with the distribution of the values for those regions, and the correlation 1332 coefficients are presented in the upper triangle boxes (B).

\section{Supplementary Figure $\mathbf{S 2}$}

1335 GSEA enrichment plots of representative gene sets from Glycolysis (Core vs. Inv) (A), 1336 Glycolysis (Rim vs. Inv) (B), Hypoxia (Core vs. Inv) (C), Hypoxia (Rim vs. Inv) (D), 1337 Inflammatory response (5ALA+ vs. 5ALA-) (E), and TNFA signaling via NFkB (5ALA+ 1338 vs. 5ALA-) (F). The heatmaps show hierarchical clustering based on z-scored 1339 expression $\left(\log _{2} T P M+1\right)$ of the leading edge genes of Glycolysis (G). Boxplot 1340 illustrating significant expression $(\mathrm{BH}<0.05)$ changes between brain regions for leading 1341 edge genes from- Hypoxia $(\mathrm{H})$, Glycolysis (I), and Inflammatory response/ TNFA 1342 signaling via NFkB (J). Hierarchical clustering heatmap of brain cell type 1343 (oligodendrocytes, neurons, astrocytes, and cultured astroglia) specific enrichment 
1344 analysis. The color intensities depict the normalized enrichment scores (NES) of each 1345 cell type on different brain regions (Core, Rim, Inv, 5ALA-, 5ALA+) (K).

\section{Supplementary Figure S3}

1348 GSEA plots of gene clusters from GBM subtypes - Classical (Core vs. 5ALA+) (A), 1349 Classical (Rim vs. 5ALA+) (B), Neural (Inv vs. Core) (C), and Proneural (5ALA-vs. $13505 A L A+)(D)$. The boxplots represent the $\log _{2}(T P M+1)$ expression levels for each brain 1351 region (Core, Rim, Inv) and 5ALA sorted cells (5ALA-, 5ALA+), with significant expression differences $(P$-value $<0.05)$ denoted among GBM regions and 5ALA sorted 1353 cells for GBM subtypes - Classical (E), Neural (F), Proneural $(G)$ and Mesenchymal $1354(\mathrm{H})$.

\section{Supplementary Figure S4}

1357 Box plots showing the relative $\log _{2}(T P M+1)$ expression levels of three cellular and 1358 metabolic state gene sets - GPM (A), MTC (B), and Inflammatory wound response (C) for Core, Rim, Inv, 5ALA- and 5ALA+ cells. Respective p-values (if $<0.05$ ) are also shown.

\section{Supplementary Figure S5}

1363 Hallmark enrichment is shown via a color code representing the difference between 1364 delta exon and intron (( $\Delta$ exon- $\Delta$ intron). Thus, positive (red) and negative (blue) values indicate higher exon and intron changes respectively in 5ALA+ cells (A). Regulated DEGs with a significant change in exon and intron counts are shown as heatmaps for TNFA signaling (B), Inflammatory response (C), Mesenchymal subtype (D), Inflammatory wound response and MTC $(F)$. The color code represents the difference between delta exon and delta intron ( $\Delta$ exon- $\Delta$ intron). The significant changes of $\Delta$ exon$\Delta$ intron are shown as $\left(^{*}\right)$. The size of the circle is according to the adjusted -log10 pvalue. 


\section{Supplementary Figure S6}

1376 Bar plots represent delta exon ( $\Delta$ exon), delta intron ( $\Delta$ intron) and difference between $1377 \Delta$ exon and $\Delta$ intron ( $\Delta$ exon - $\Delta$ intron) for the significantly DEGs. The circles adjacent to 1378 the gene symbol represent the gene-signature and the colors of the circle indicate 1379 different gene-signatures. [TF $=$ Transcription Factor; Inf. wound response = 1380 Inflammatory Wound Response; TNFA = tumor necrosis factor - alpha; MTC = 1381 Mitochondrial Subtype].

\section{Supplementary Figure S7}

1384 Correlation between mRNAsi values and mRNA expression for available Malta et al. 1385 stem cell markers (A). Correlation between mRNAsi and mRNA expression for markers 1386 from stem cell marker database (B). Heatmap of RNA-seq expression z-scores for the 1387 Malta et al. stem cell marker signatures (C) and markers from stem cell marker 1388 database (D). Heatmap illustrating normalized enrichment scores (NES) of GSEA with 1389 padj $<0.05$ for selected stemness gene-sets from different studies $(E)$.

\section{Supplementary Figure S8}

1392 Kaplan-Meier survival analysis for disease free survival curves based on median (low expression and high expression) of five different gene signatures from TCGA-GBM samples. High TNFA leading edge signature gene expression indicated poor survival in

1395 TNFA signaling $(P=0.028)(A)$. High expression of Inflammatory response $(P=0.0045)$ $(B)$, Mesenchymal $(0.0014)(C)$, Inflammatory wound response $(P=0.0086)(D)$ and GPM (0.045) (E) gene signatures exhibited poor survival respectively.

\section{Supplementary Figure S9}

1400 Hallmark enrichment analysis of DEGs between recurrent and primary GBM tumors.

1401 The hallmarks that are enriched in recurrent tumors in comparison to parimary tumors 1402 from TCGA, CCGA and GLASS cohorts are illustrated. Each bubble indicate one 1403 particular hallmark (y-axis). The $x$-axis indicate a $-\log 10$ adjusted $p$-value. The color 1404 gradiant of the bubble indicates the normalized enrichment score (NES) whereas the 
1405 size of the bubble depends on the gene-count for each hallmark. Paired and unpaired 1406 analysis was performed for the TCGA cohort.

1407 Supplementary Tables

1408 Supplementary Table 1: Clinical, pathological and molecular characterization of GBM 1409 patients, and compiled Gene-signatures

1410 Supplementary Table 2: Hallamark GSEA and leading gene-edge gene list

1411 Supplementary Table 3: Neural cell type GSEA and leading gene-edge gene list

1412 Supplementary Table 4: GBM-subtype GSEA and leading gene-edge gene list

1413 Supplementary Table 5: Cellular and metabolic gene-set GSEA and leading gene-

1414 edge gene list

1415 Supplementary Table 6: TF-target gene interaction

1416 Supplementary Table 7: Enrichment of selected gene-signatures in DEGs with 1417 significantly altered exon and intron counts in 5ALA+ cells

1418 Supplementary Table 8: Enrichment of stemness associated gene-signatures

1419 Supplementary Table 9: Genes correlated with stemness in 5ALA+ cells

1420 Supplementary Table 10: Genes associated with poor survival

1421 Supplementary Table 11: GSEA in primary and recurrent tumors 
Figure 1

A
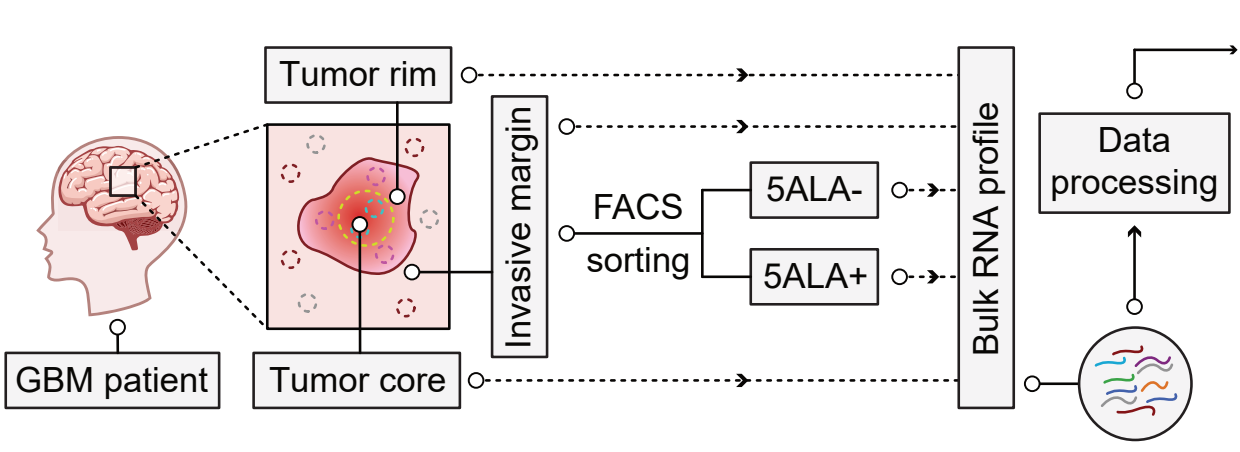

\begin{tabular}{|c|c|c|}
\hline \multicolumn{3}{|c|}{ Spatial transcriptomics data analysis } \\
\hline Clustering & GSEA & Volcano plot \\
\hline \hline & & \\
\hline Sample & \\
\hline EISA & Network & OCLR \\
\hline
\end{tabular}

B

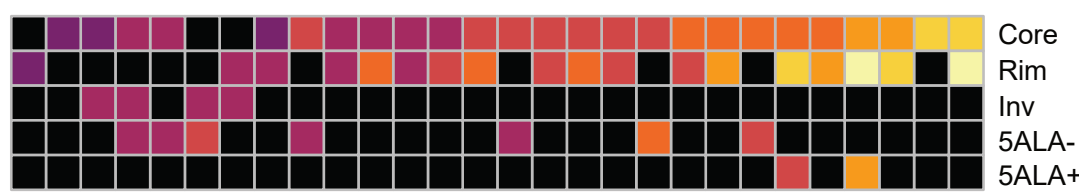

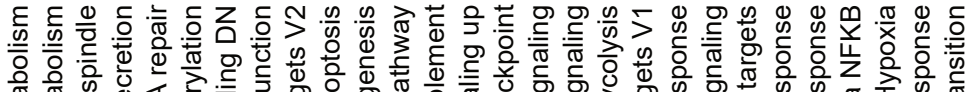

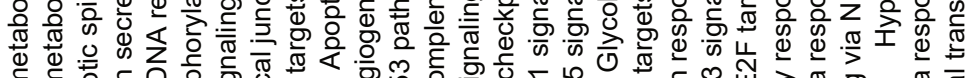

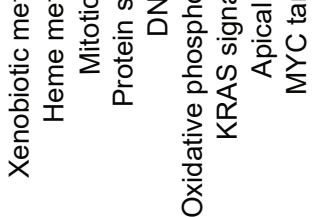

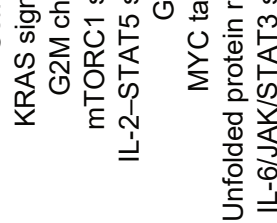

NES

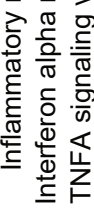

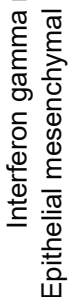

C

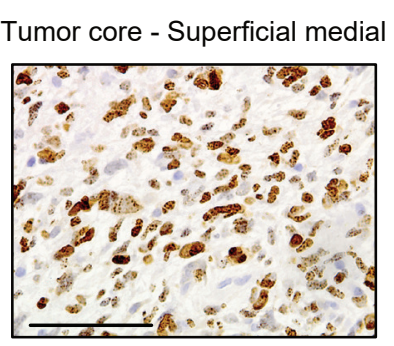

E

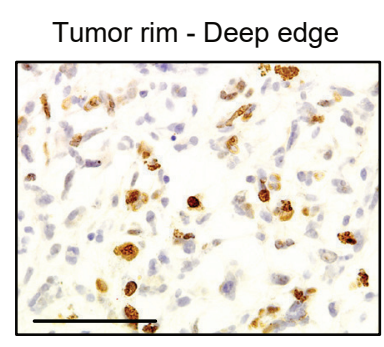

D Tumor core - Anterior medial

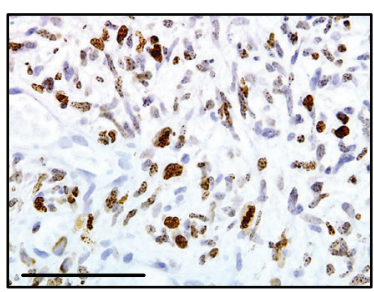

$\mathbf{F}$

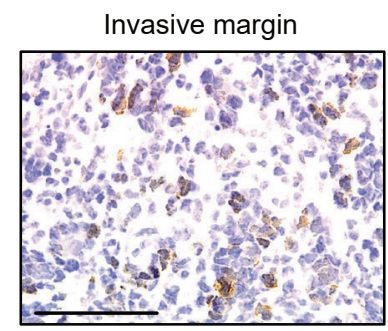

G

Tumor core - High vascularity

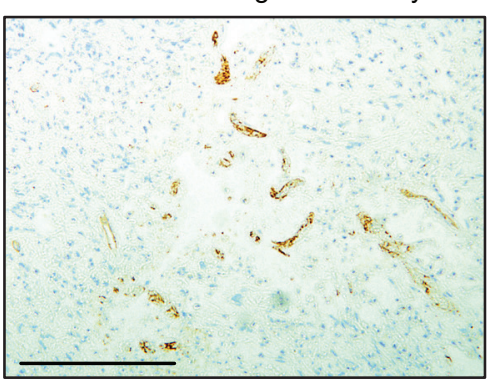

H

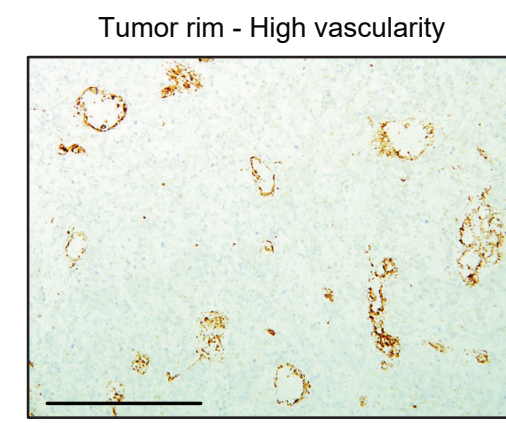

Invasive margin - Low vascularity

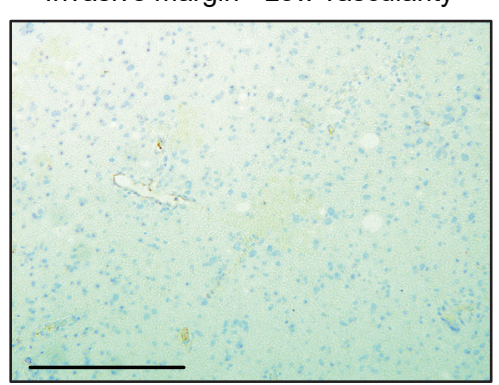

\section{Hypoxia}

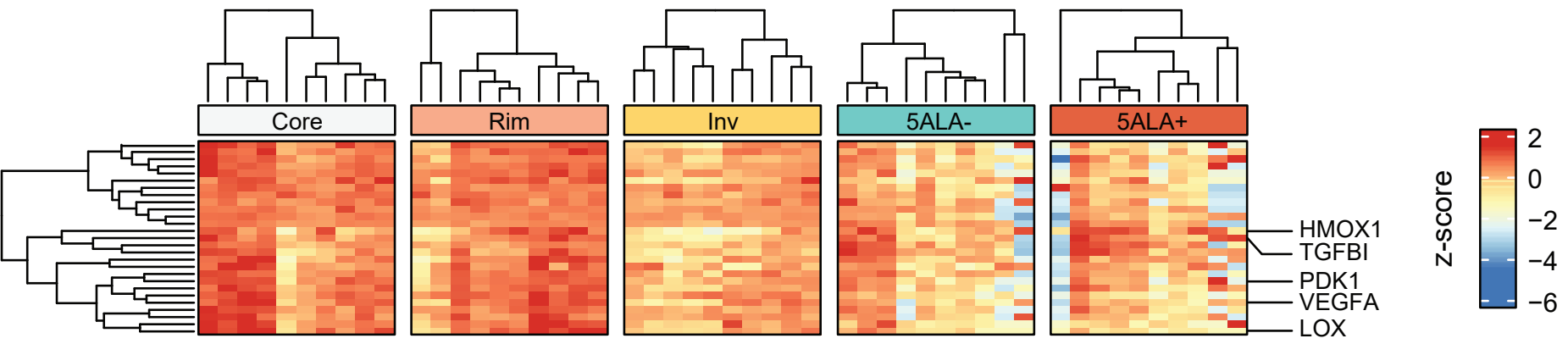

Inflammatory response/ TNFA signaling via NFKB

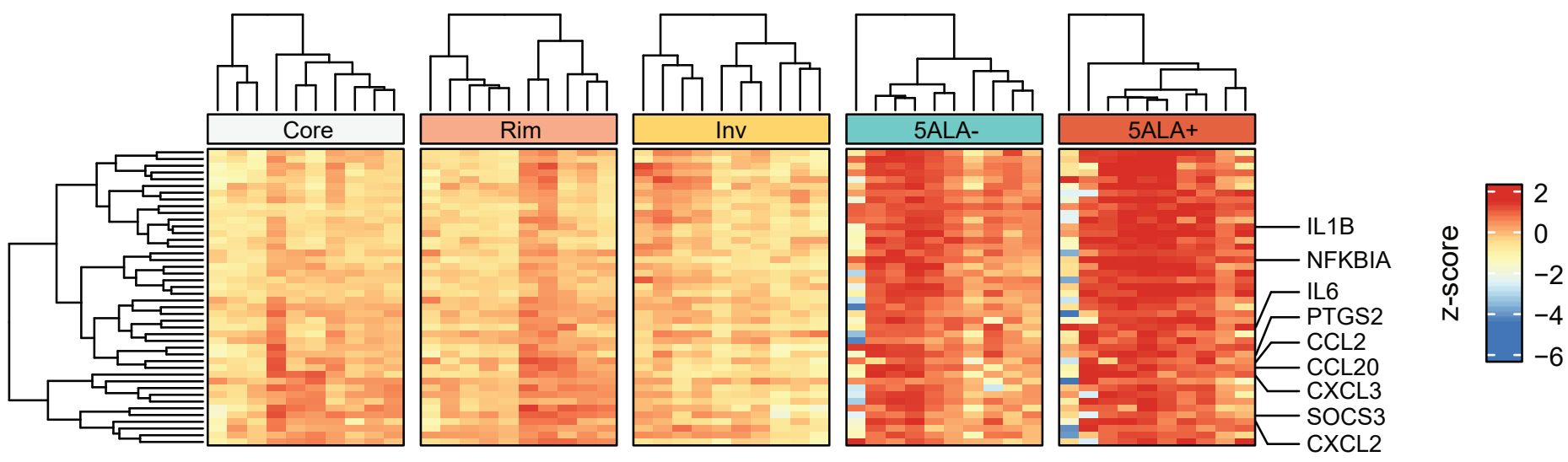


Figure 2

A

GBM subtypes

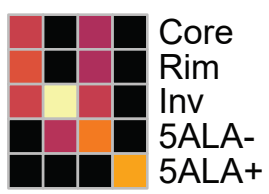

$\overline{\widetilde{\sigma}} \overline{\mathrm{T}} \overline{\mathrm{\sigma}} \overline{\mathrm{\sigma}}$

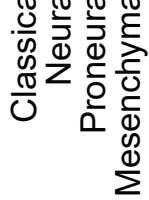

NES

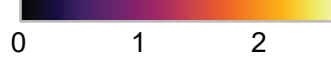

$\mathbf{F}$

B 5ALA+ vs 5ALA- (Mesenchymal)

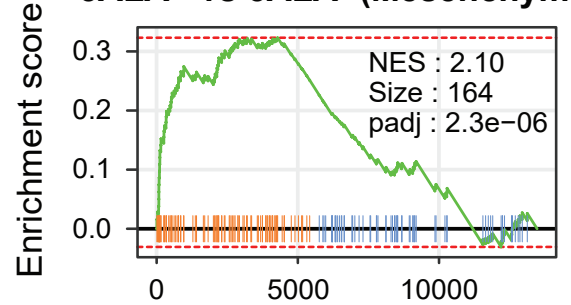

Position of ranked list of genes

D

Tumor rim

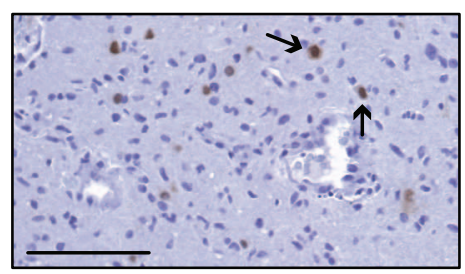

Classical
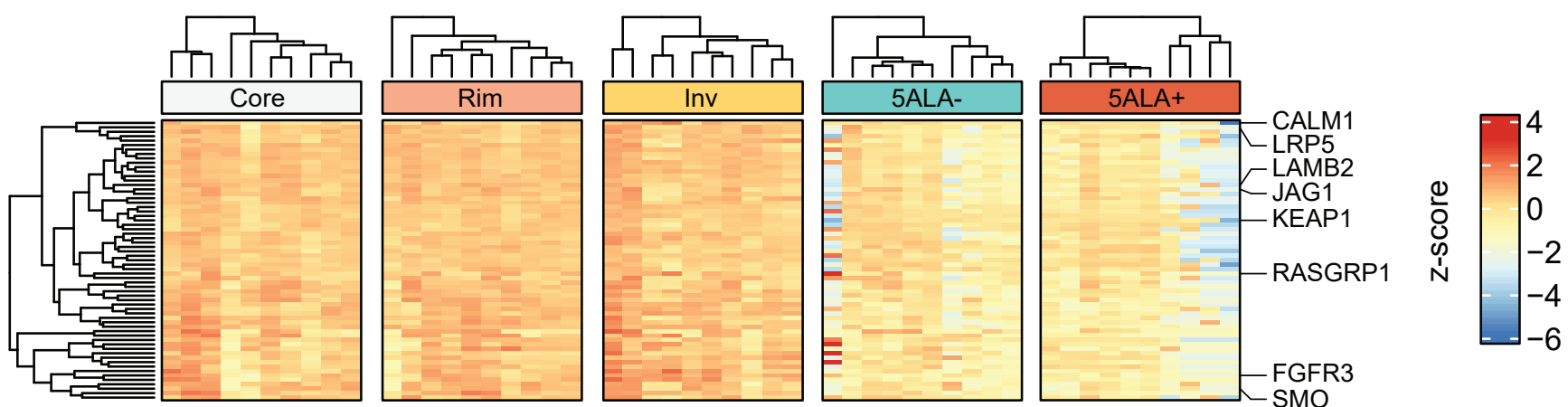

G
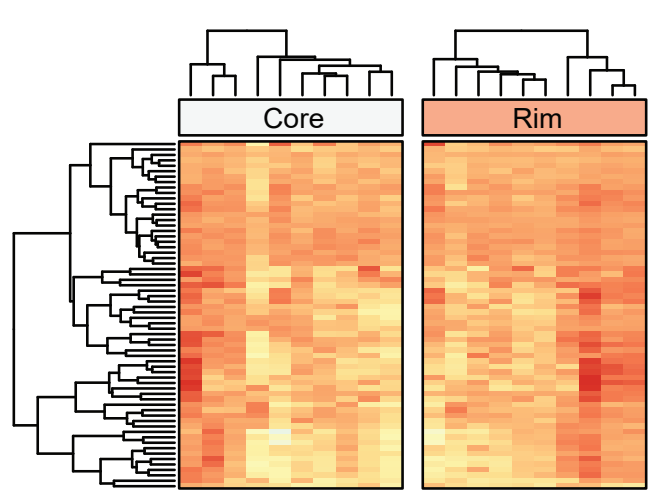

Neural
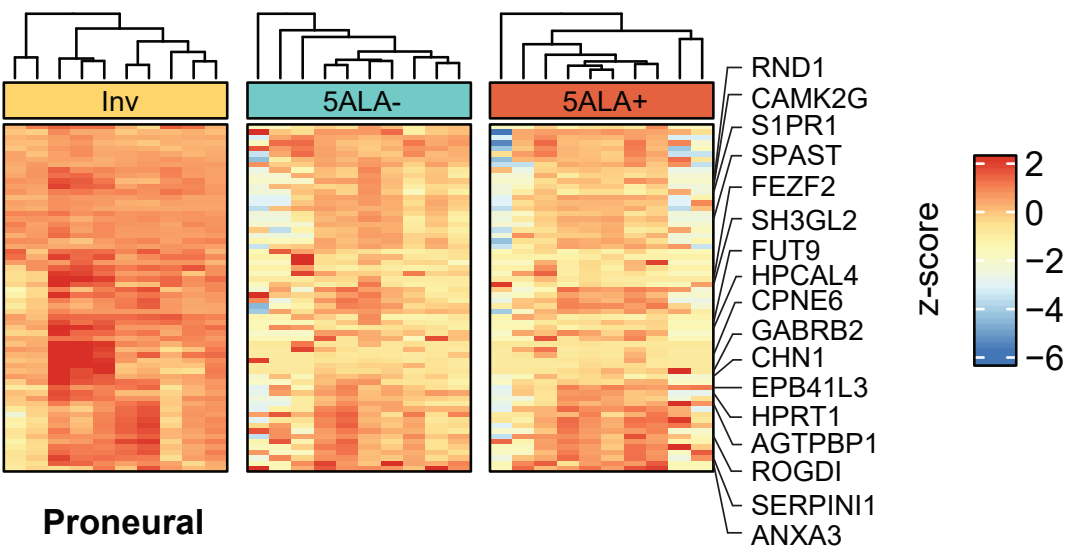

H

Proneural
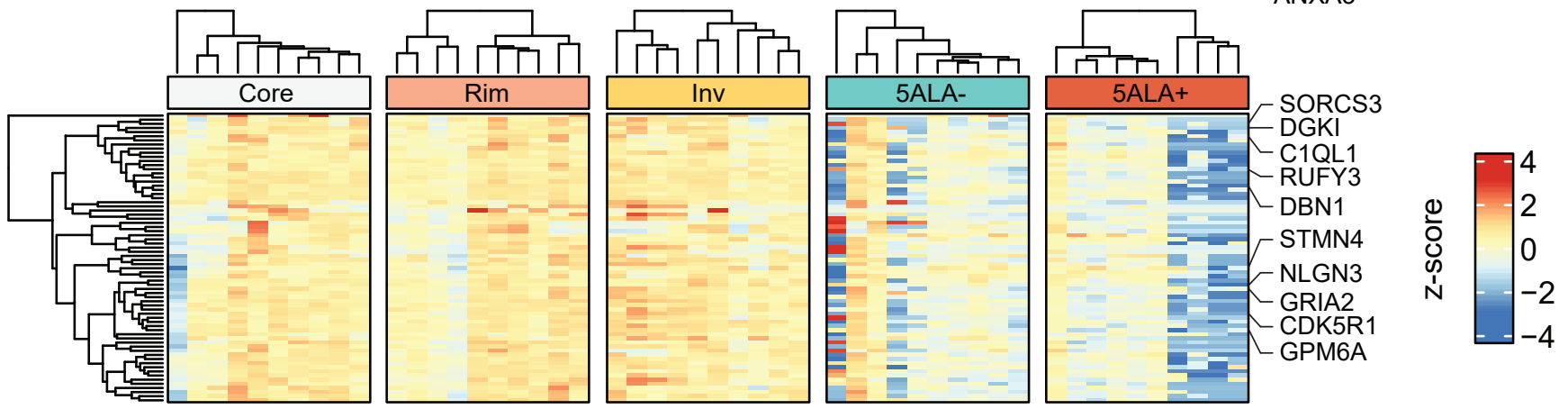

I

Mesenchymal
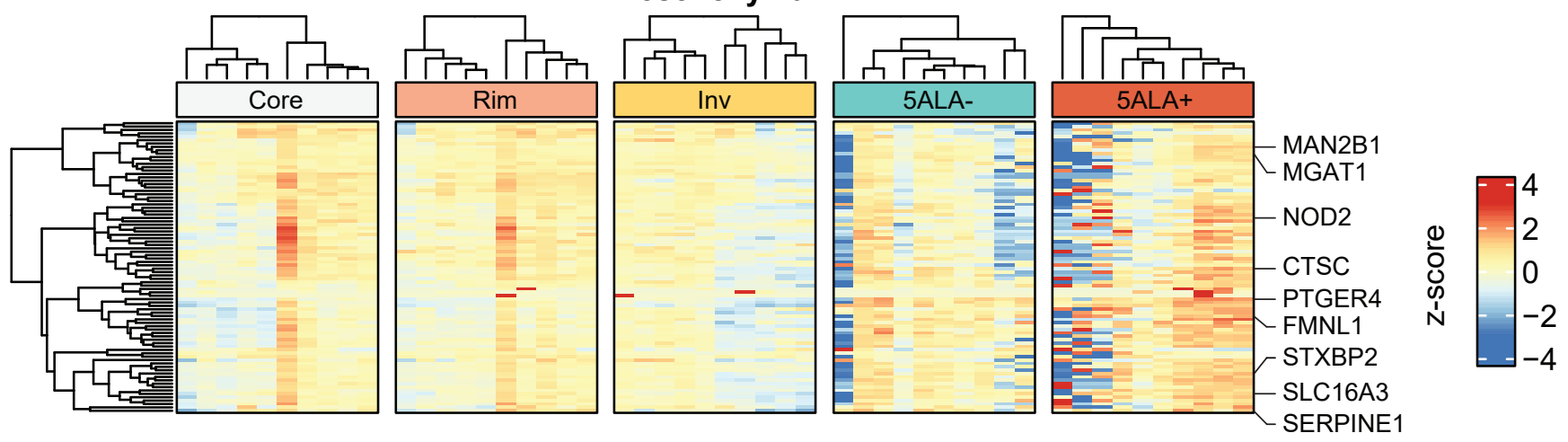


\section{Figure 3}

A

Cellular and metabolic states

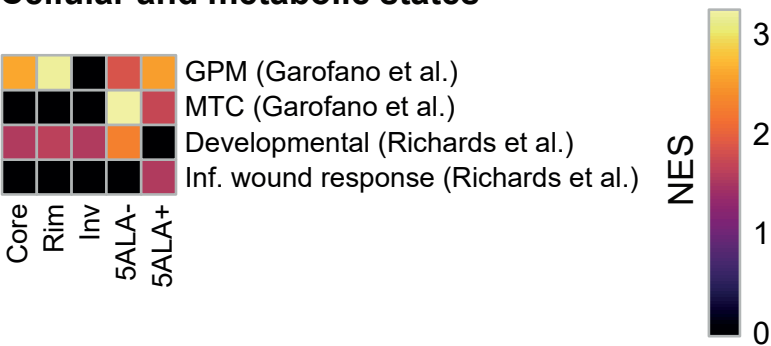

C

E

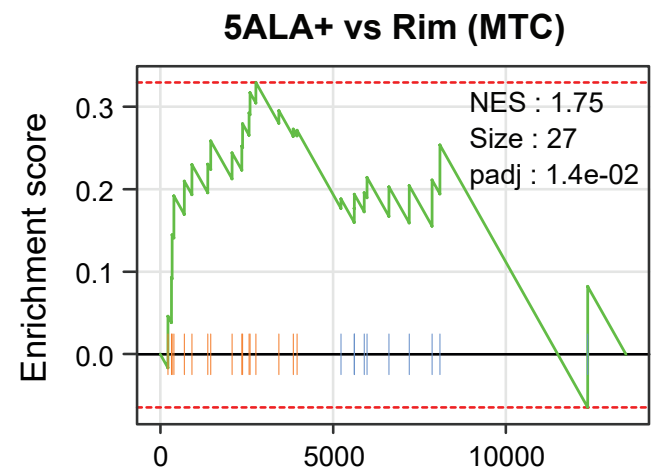

GPM

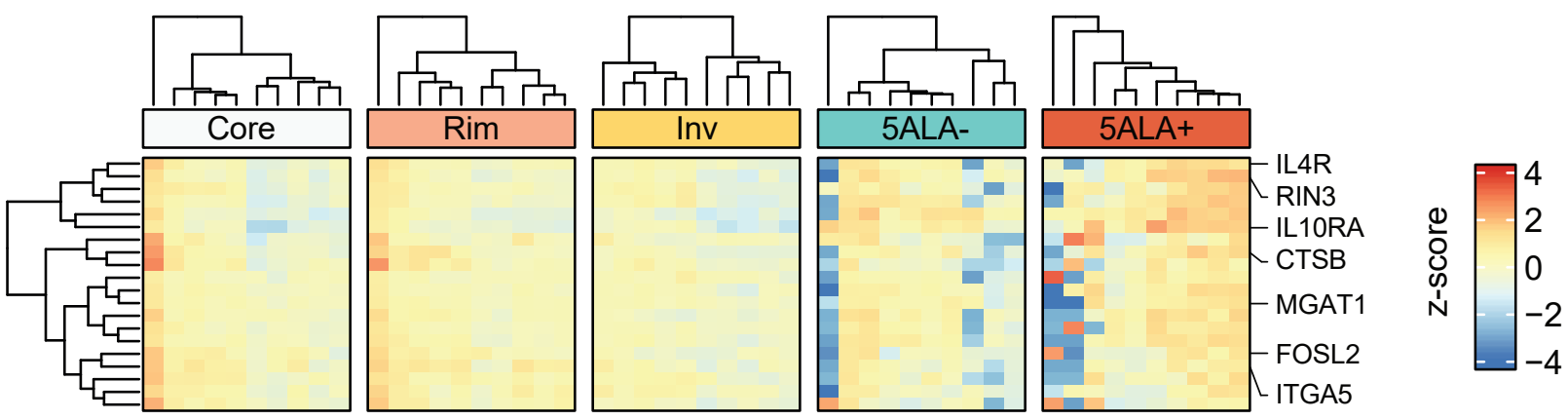

F

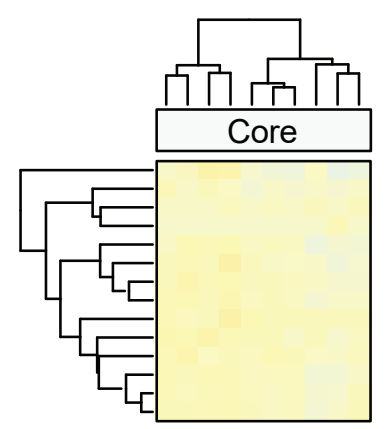

G

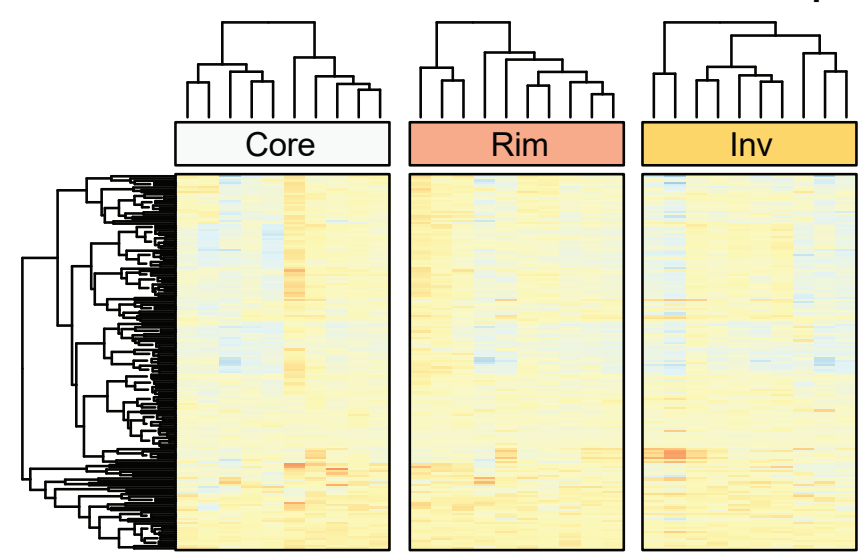

B

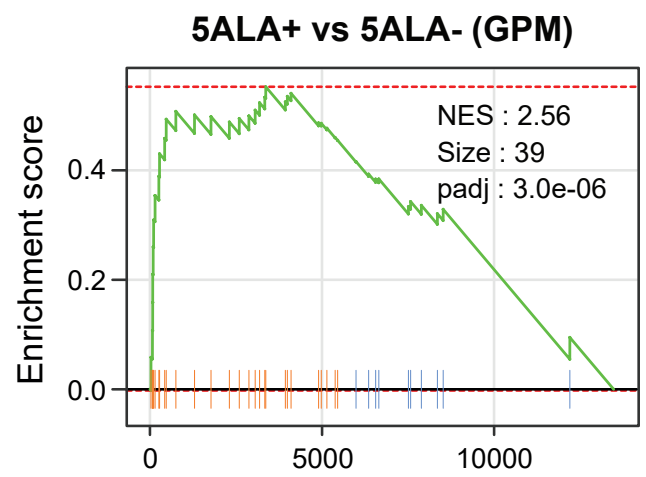

D

5ALA+ vs Inv (Inf. wound response)

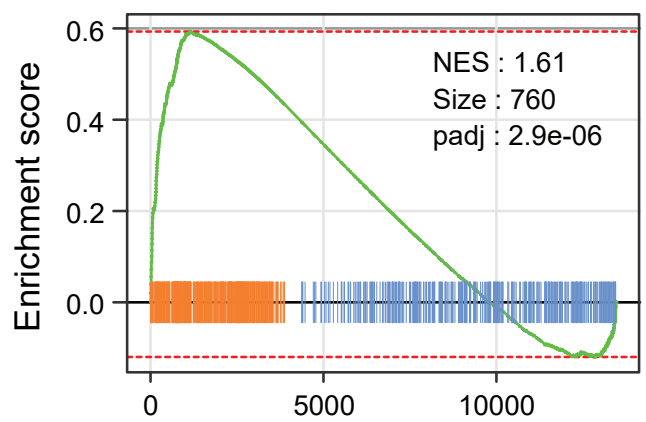




\section{Figure 4}

A

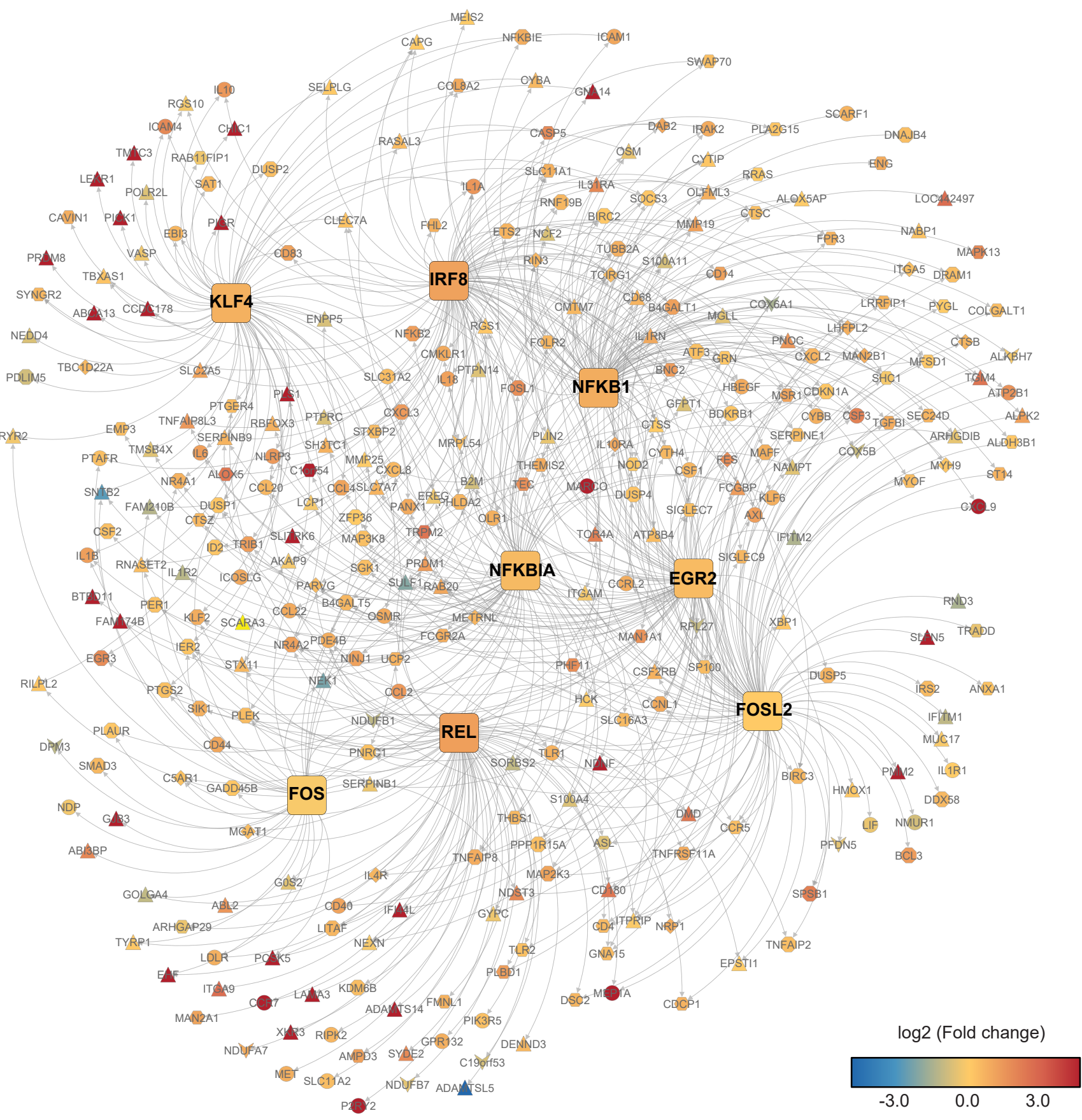

$\square$ Transcription factor $\bigcirc$ Inflammatory response $\triangle$ Inf. wound response $V$ MTC $\square$ Mesenchymal $\diamond$ GPM $\bigcirc$ TNFA signaling

B
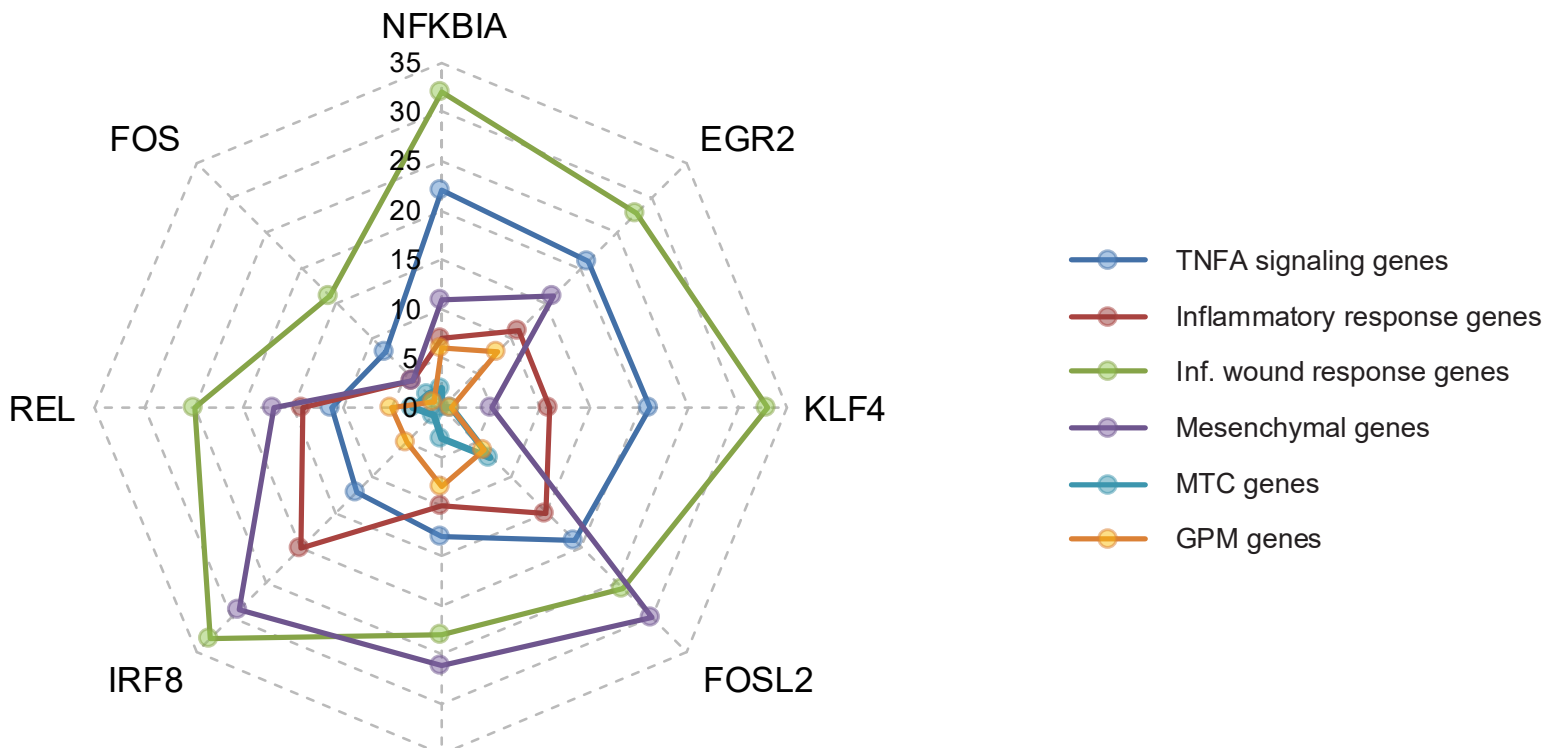


\section{Figure 5}

A

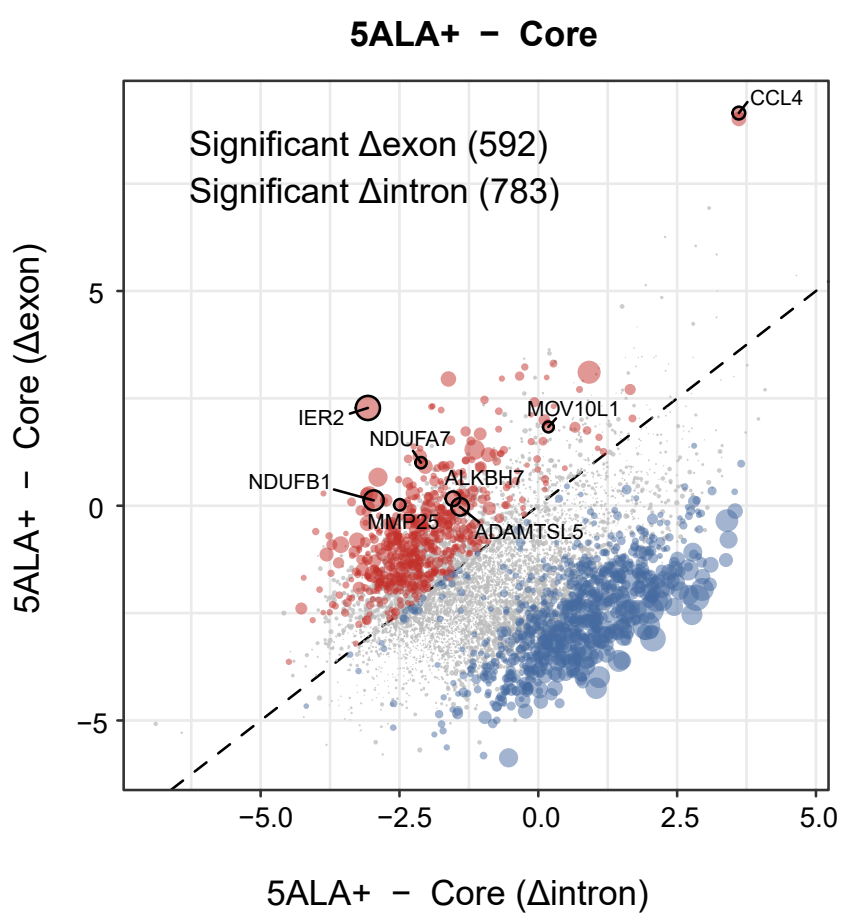

C

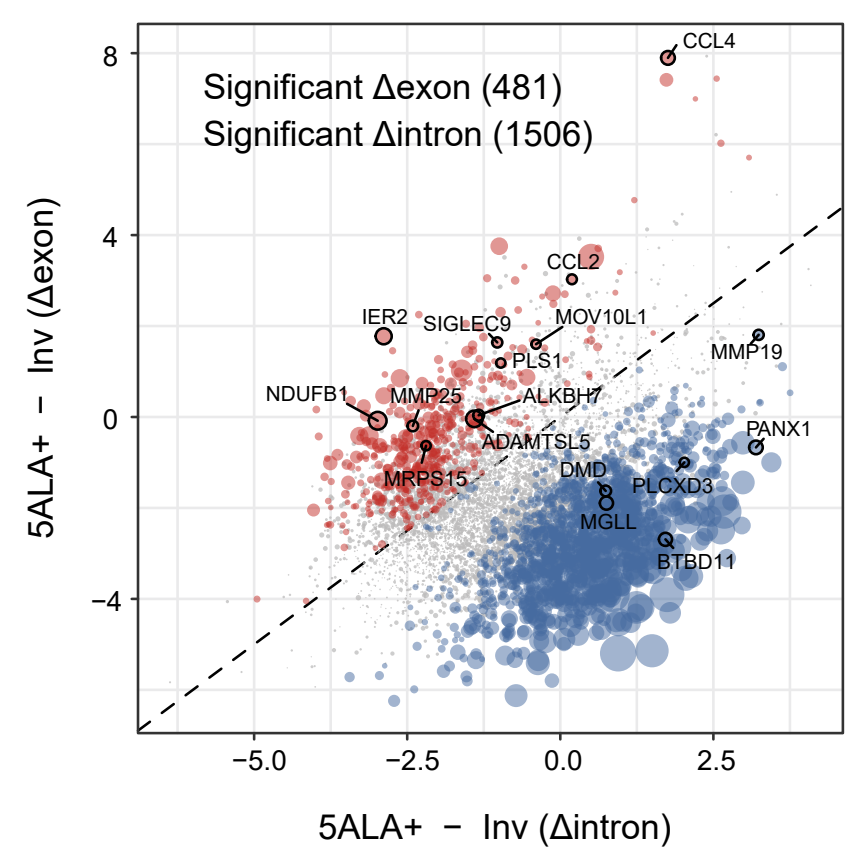

B

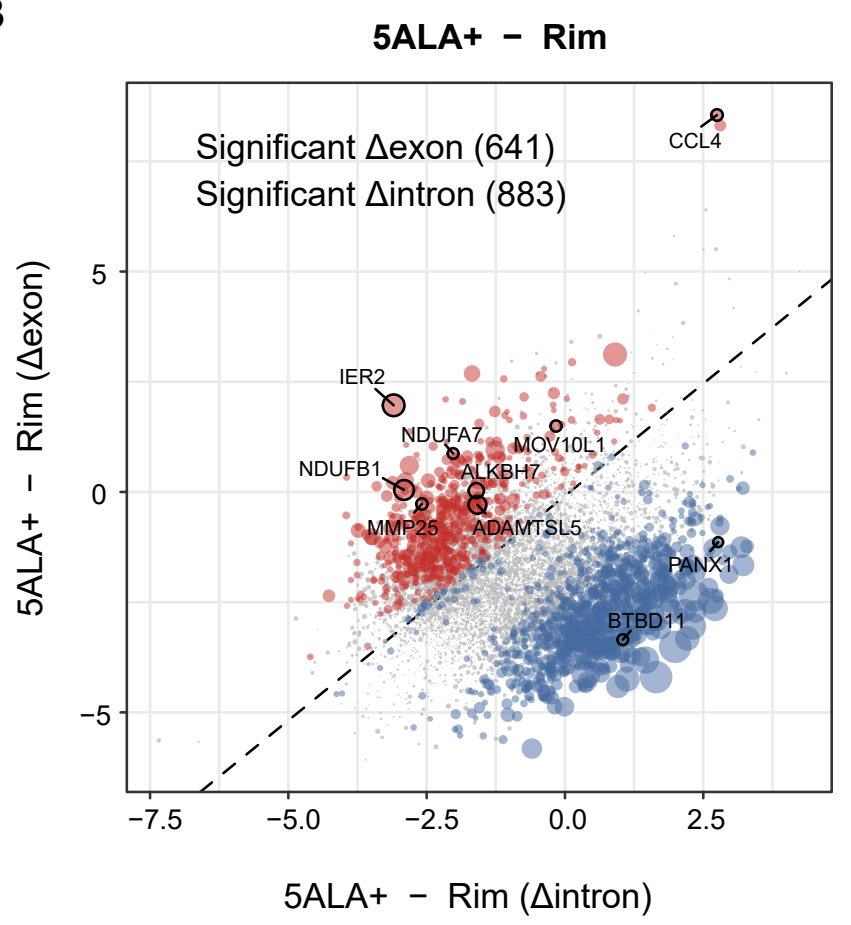

D

5ALA+ - 5ALA-

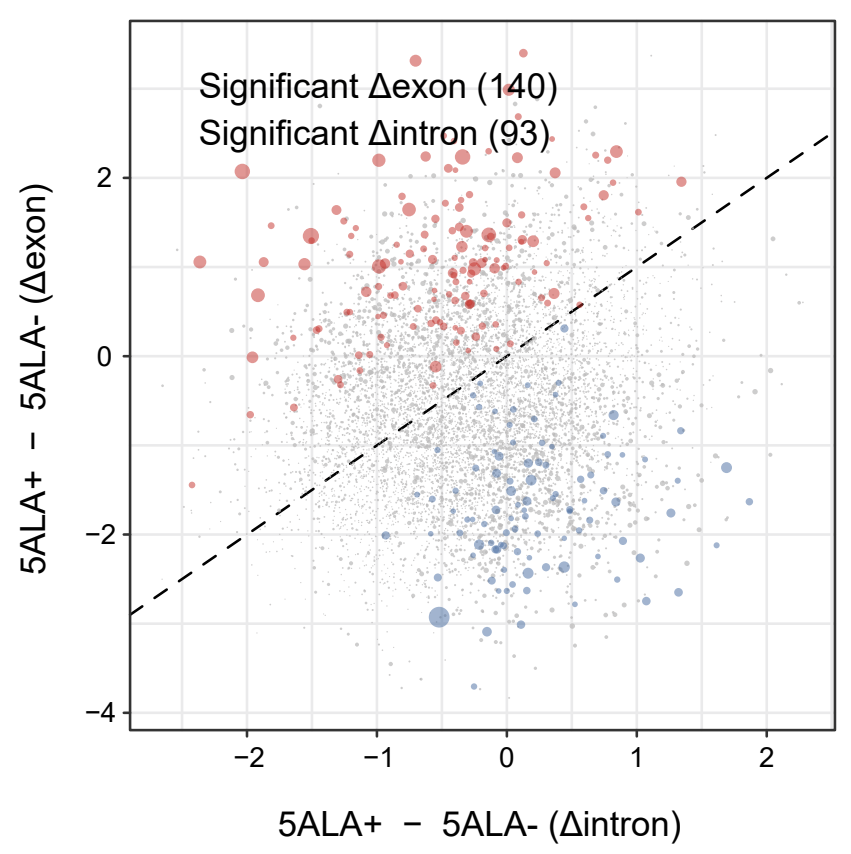

E

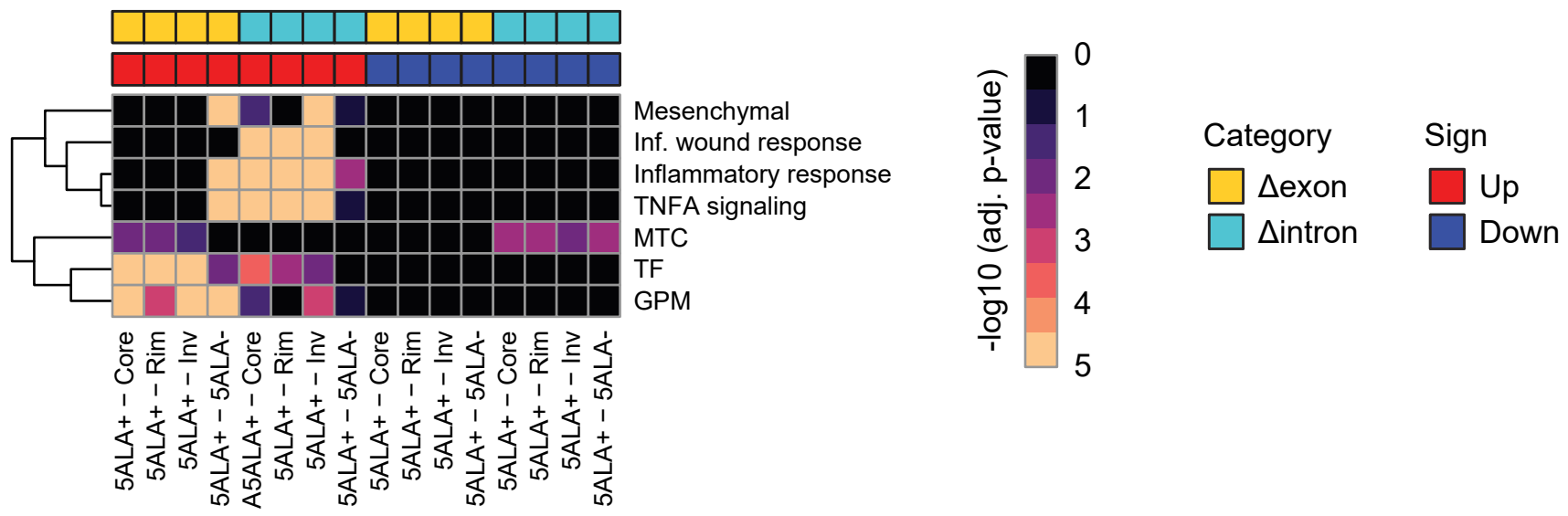




\section{Figure 6}

A

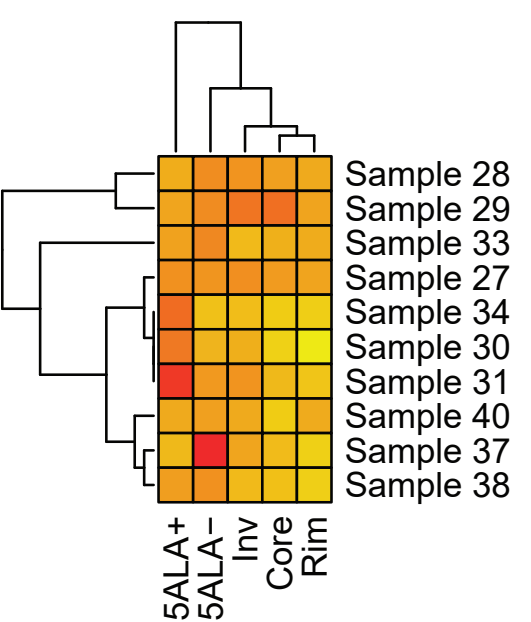

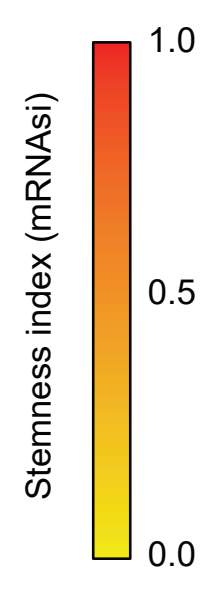

B

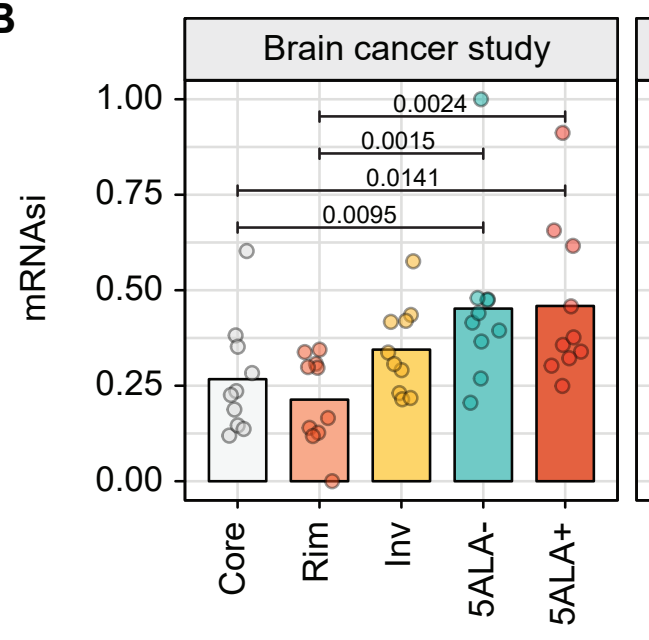

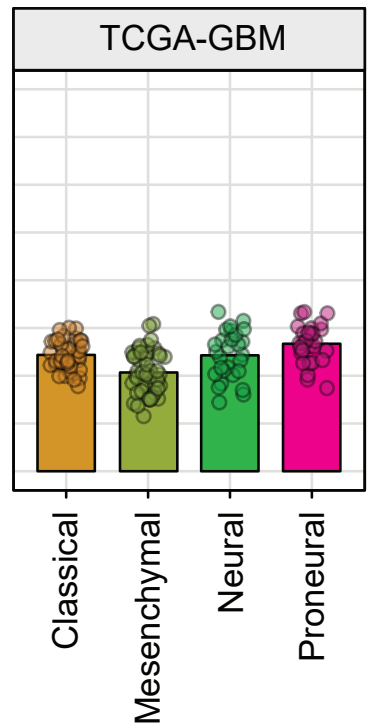

C
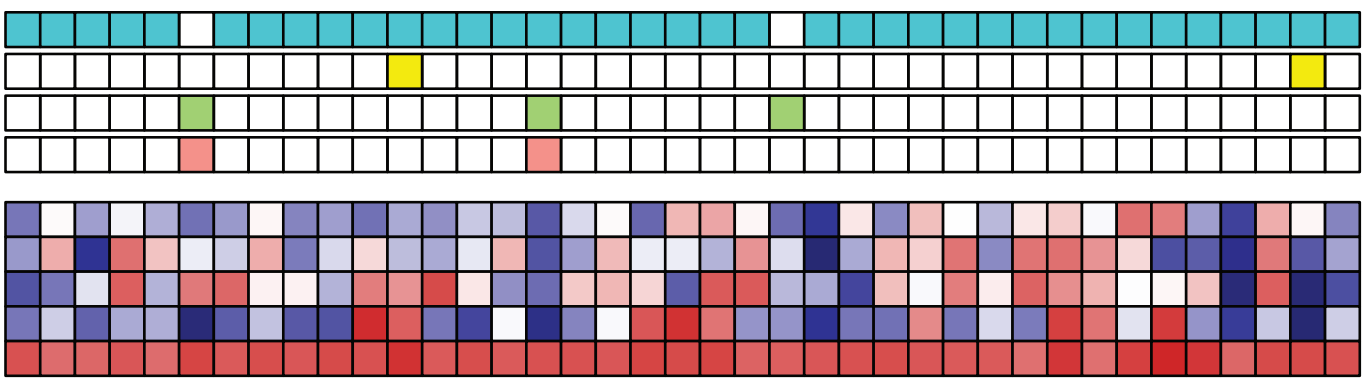

Core

Rim

Inv

$5 A L A-$

5ALA+

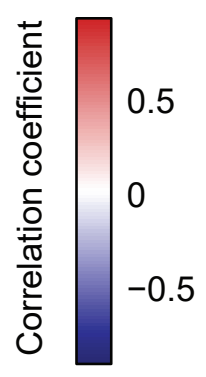

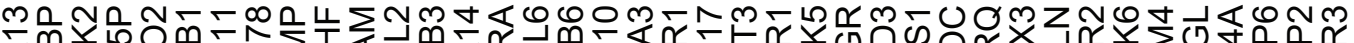

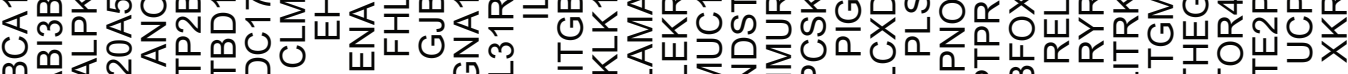

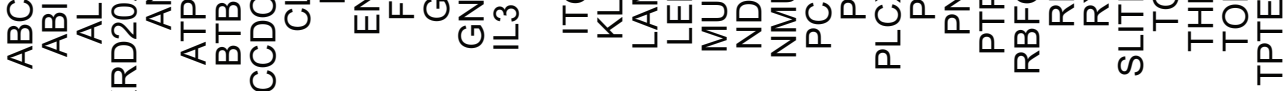

文

$\square$ Inf. wound response $\square$ Inflammatory response $\square$ Mesenchymal $\square$ TNFA signaling

D
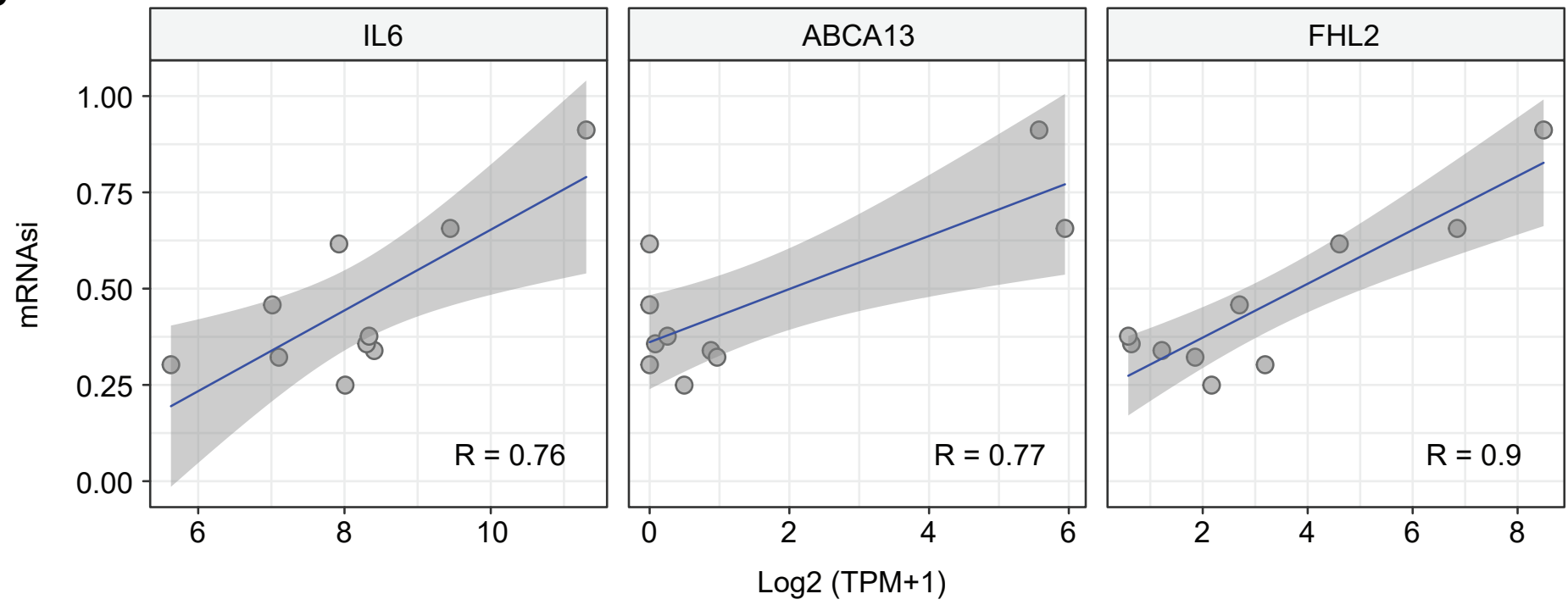

E

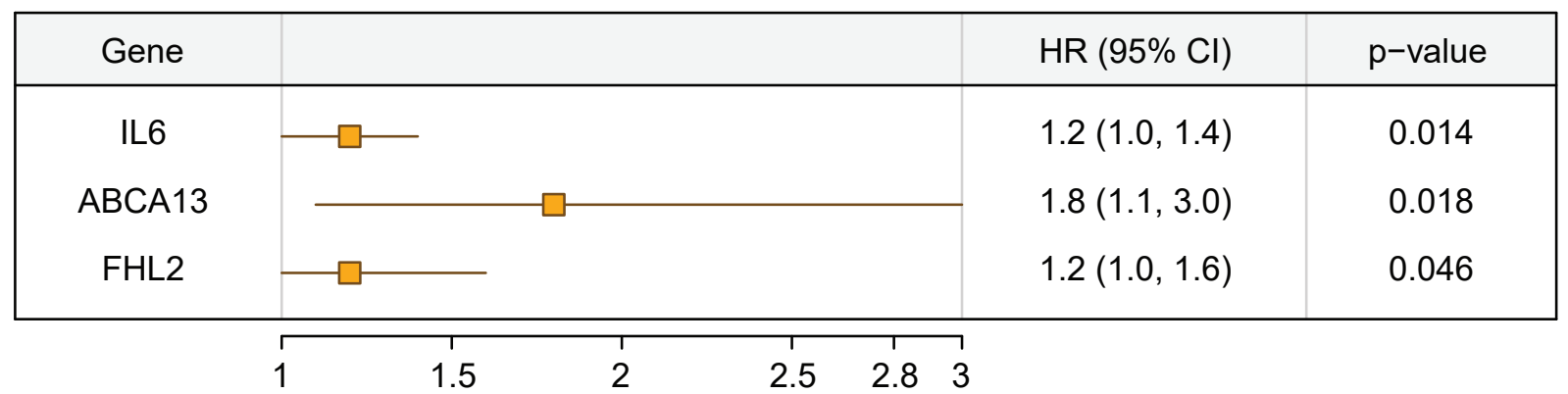


A

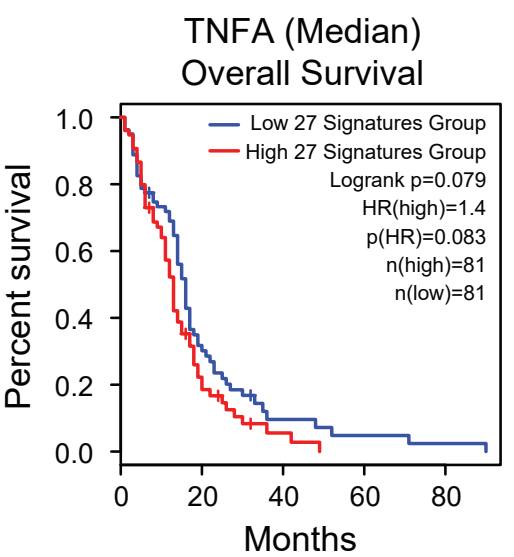

D

Inf. wound response (Median)

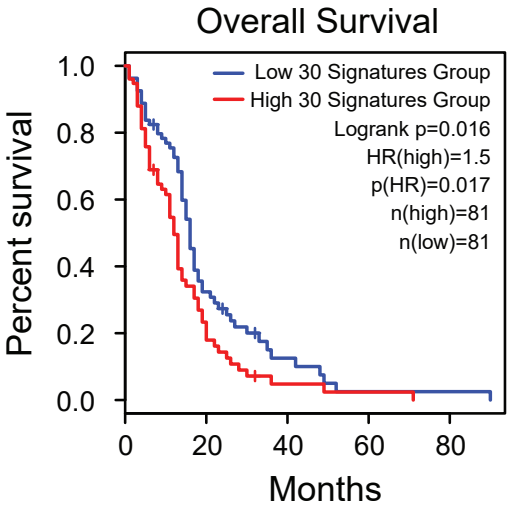

G

TCGA - Recurrent tumor (5ALA+ score vs. survival)

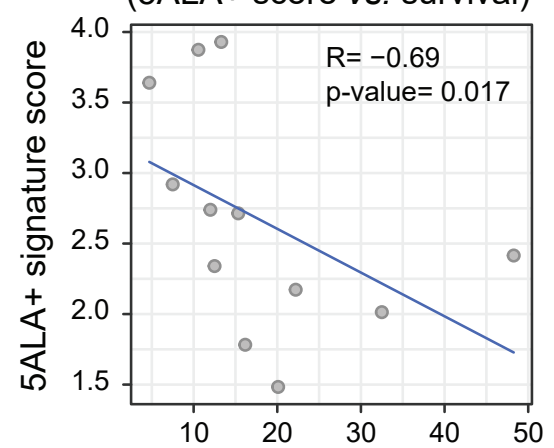

Overall survival (month)

GLASS - Recurrent tumor (5ALA+ score vs. survival)

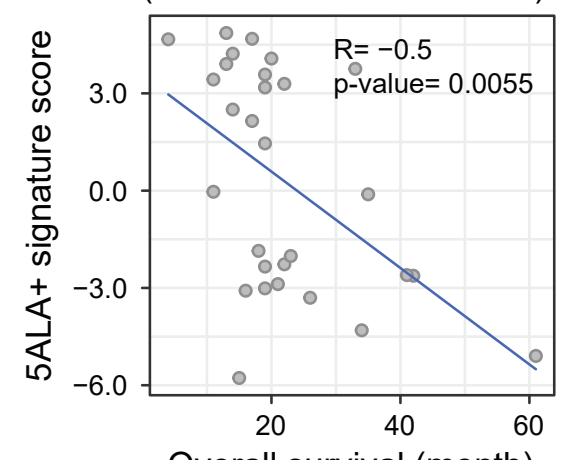

K
B Inflammatory response (Median) C Overall Survival

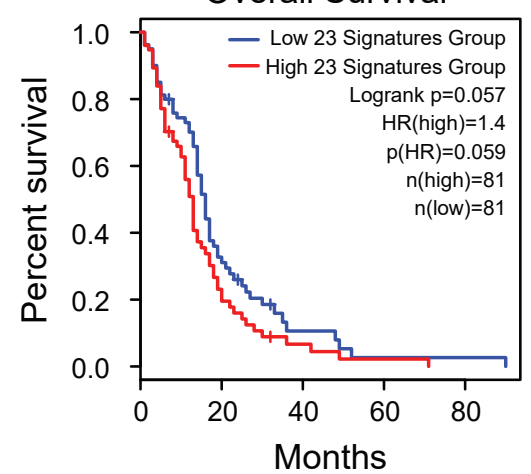

$E$

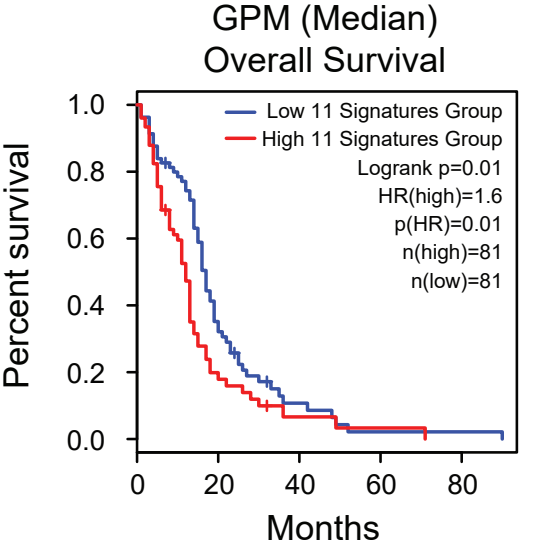

H

TCGA - Primary tumor (5ALA+ score vs. survival)

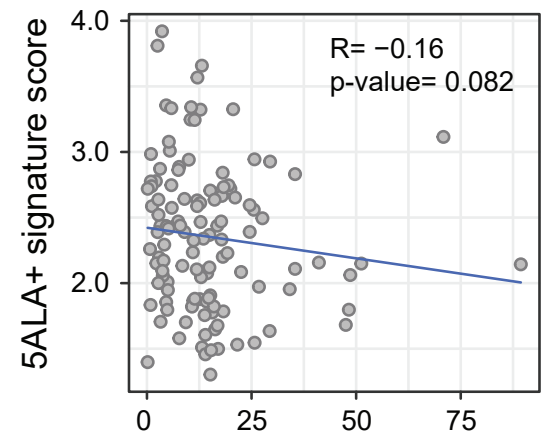

Overall survival (month)

J GLASS - Primary tumor (5ALA+ score vs. survival)

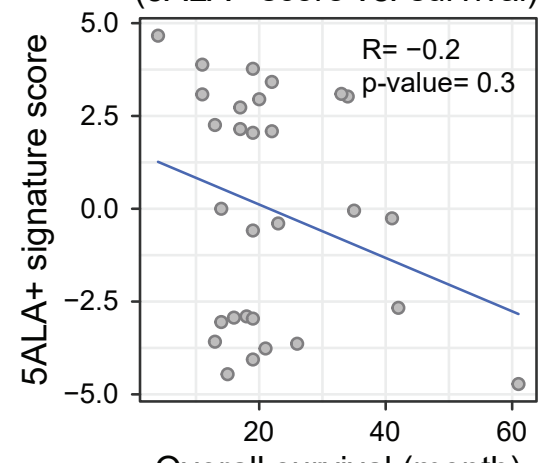

Mesenchymal (Median) Overall Survival

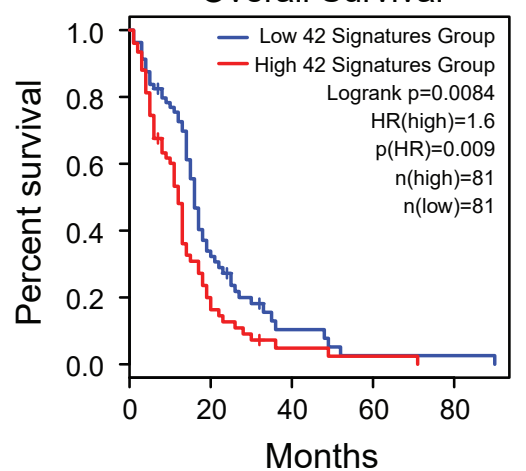

$\mathbf{F}$
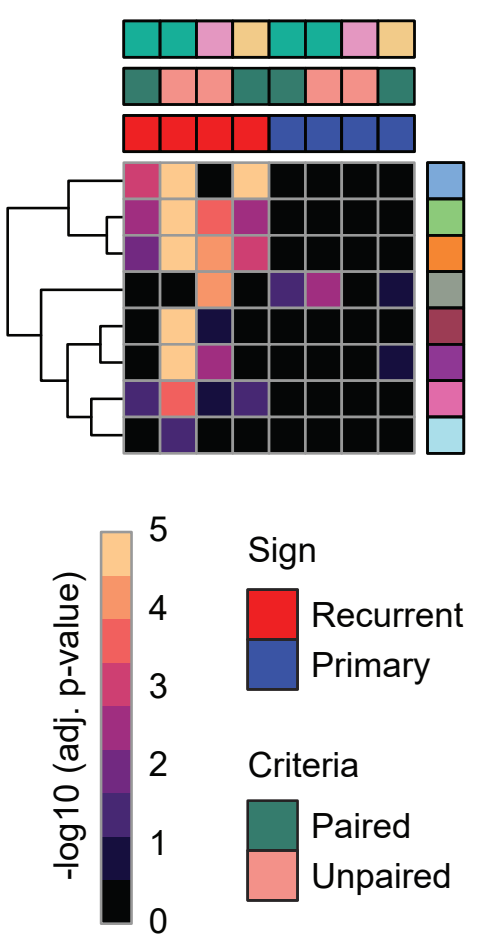

Datasets

\begin{tabular}{|l|l}
\hline TCGA \\
CGGA \\
\hline GLASS
\end{tabular}

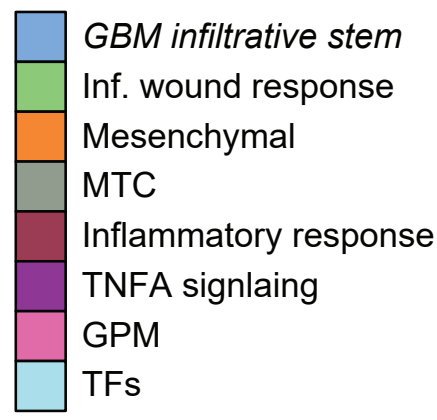

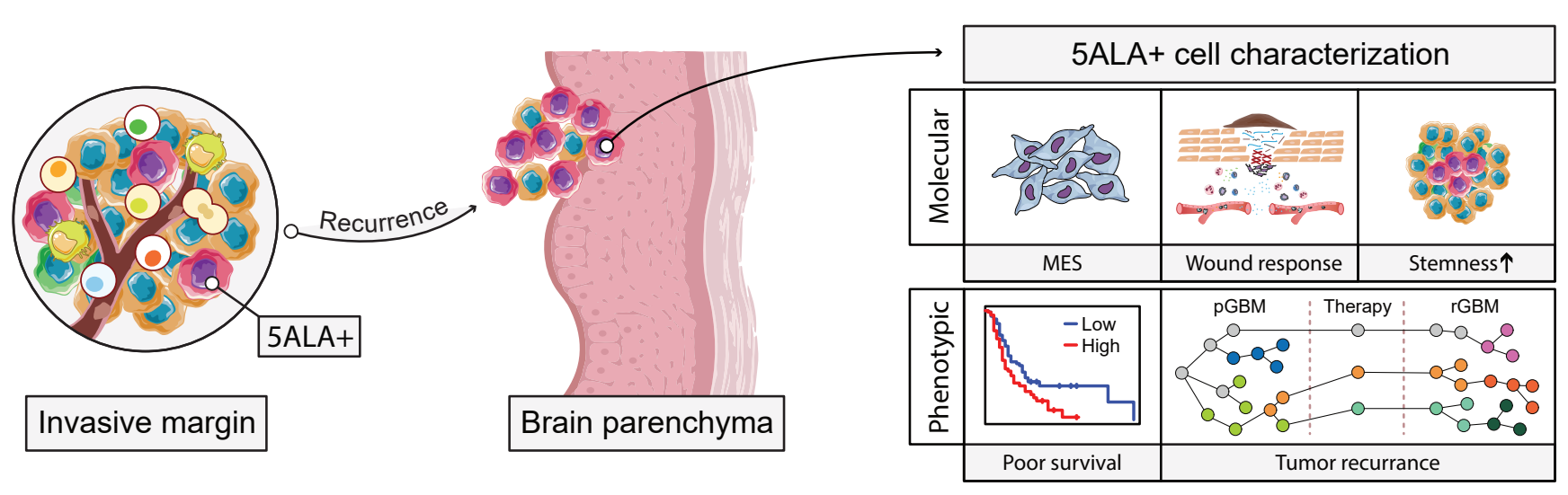




\section{Supplementary Files}

This is a list of supplementary files associated with this preprint. Click to download.

- SupplementaryTables.zip

- MergedSuppFigures04.08.2021.pdf 